\title{
Deep learning-based electrocardiogram rhythm and beat features for heart abnormality classification
}

\author{
Annisa Darmawahyuni ${ }^{1}$, Siti Nurmaini ${ }^{\text {Corresp., } 1}{ }^{1}$, Muhammad Naufal Rachmatullah ${ }^{1}$, Bambang Tutuko ${ }^{1}$, Ade Iriani \\ Sapitri $^{1}$, Firdaus Firdaus ${ }^{1}$, Ahmad Fansyuri ${ }^{1}$, Aldi Predyansyah ${ }^{1}$ \\ 1 Intelligent System Research Group, Faculty of Computer Science, Sriwijaya University, Palembang, 30139, Indonesia \\ Corresponding Author: Siti Nurmaini \\ Email address: siti_nurmaini@unsri.ac.id
}

Background. Electrocardiogram (ECG) signal classification plays a critical role in the automatic diagnosis of heart abnormalities. While most ECG signal patterns cannot be recognized by human interpreter, can be detected with precision using artificial intelligence approaches, making the ECG a powerful non-invasive biomarker. However, performing rapid and accurate ECG signal classification is difficult due to the low amplitude, complexity, and non-linearity. The widely available and proposed deep learning (DL) method has explored and presented an opportunity to substantially improve the accuracy of automated ECG classification analysis using rhythm or beat feature. Unfortunately, a comprehensive and general evaluation of the specific DL architecture for ECG analysis across a wide variety of rhythm and beat features has not been previously reported. Some previous studies have been concerned with detecting ECG class abnormalities only through rhythm or beat feature separately.

Methods. This study proposes a single architecture based on DL method with one-dimensional convolutional neural network (1D-CNN) architecture, to automatically classify 24 patterns of ECG signals through both features rhythm and beat. To validate the proposed model, five databases which consisted of nine-class of ECG-base rhythm and 15-class of ECG-based beat are utilized in this study. The proposed DL network applied and experimented with varying datasets with different frequency samplings in intra and inter-patient scheme.

Results. Using a 10-fold cross-validation scheme, the performance results had an accuracy of 99.98\%, a sensitivity of $99.90 \%$, a specificity of $99.89 \%$, a precision of $99.90 \%$, and an F1-score of $99.99 \%$ for ECG rhythm classification. Also, for ECG beat classification, the model obtained an accuracy of $99.87 \%$, a sensitivity of $96.97 \%$, a specificity of $99.89 \%$, a precision of $92.23 \%$, and an F1-score of $94.39 \%$. In conclusion, this study provides clinicians with an advanced methodology for detecting and discriminating heart abnormalities between different ECG rhythm and beat assessments by using one outstanding proposed DL architecture. 


\title{
1 Deep Learning-based electrocardiogram rhythm and
} 2 beat features for heart abnormality classification

3

4

\author{
Annisa Darmawahyuni, Siti Nurmaini, Muhammad Naufal Rachmatullah, Bambang Tutuko, \\ Ade Iriani Sapitri, Firdaus Firdaus, Ahmad Fansyuri, and Aldi Predyansyah \\ Intelligent System Research Group, Universitas Sriwijaya, Palembang 30139, Indonesia \\ Corresponding Author: \\ Siti Nurmaini \\ Jalan Srijaya Negara, Palembang, South Sumatera, 30139, Indonesia \\ Email address: sitinurmaini@gmail.com, siti_nurmaini@unsri.ac.id
}

\section{Abstract}

Background. Electrocardiogram (ECG) signal classification plays a critical role in the automatic diagnosis of heart abnormalities. While most ECG signal patterns cannot be recognized by human interpreter, can be detected with precision using artificial intelligence approaches, making the ECG a powerful non-invasive biomarker. However, performing rapid and accurate ECG signal classification is difficult due to the low amplitude, complexity, and non-linearity. The widely available and proposed deep learning (DL) method has explored and presented an opportunity to substantially improve the accuracy of automated ECG classification analysis using rhythm or beat feature. Unfortunately, a comprehensive and general evaluation of the specific DL architecture for ECG analysis across a wide variety of rhythm and beat features has not been previously reported. Some previous studies have been concerned with detecting ECG class abnormalities only through rhythm or beat feature separately.

Methods. This study proposes a single architecture based on DL method with one-dimensional convolutional neural network (1D-CNN) architecture, to automatically classify 24 patterns of ECG signals through both features rhythm and beat. To validate the proposed model, five databases which consisted of nine-class of ECG-base rhythm and 15-class of ECG-based beat are utilized in this study. The proposed DL network applied and experimented with varying datasets with different frequency samplings in intra and inter-patient scheme.

Results. Using a 10-fold cross-validation scheme, the performance results had an accuracy of $99.98 \%$, a sensitivity of $99.90 \%$, a specificity of $99.89 \%$, a precision of $99.90 \%$, and an F1-score of $99.99 \%$ for ECG rhythm classification. Also, for ECG beat classification, the model obtained an accuracy of $99.87 \%$, a sensitivity of $96.97 \%$, a specificity of $99.89 \%$, a precision of $92.23 \%$, and an F1-score of $94.39 \%$. In conclusion, this study provides clinicians with an advanced methodology for detecting and discriminating heart abnormalities between different ECG rhythm and beat assessments by using one outstanding proposed DL architecture. 
40

41

42

43

44

45

46

47

48

49

50

51

52

53

54

55

56

57

58

59

60

61

62

63

64

65

66

67

68

69

70

71

72

73

74

75

76

77

78

79

\section{Introduction}

Globally, heart abnormality deaths are projected to increase to 23.4 million, comprising $35 \%$ of all deaths in 2030 (World Health Organization 2016). The clinical symptoms, electrocardiogram (ECG) pattern analysis, and measurement of important cardiac biomarkers are the current heart diagnostic cornerstone (O'Gara et al. 2013). However, such diagnosis is based on the invasive laboratory test and requires specific tools, cost, and infrastructures, such as trained clinical staff for inspecting blood and performing assays and a hematology analyser with biochemical reagents (Cho et al. 2020). For this reason, such assessments are difficult to use in remote healthcare monitoring or developing countries (Makimoto et al. 2020). Analysis of ECG patterns could help with early detection of life-threatening heart abnormalities and is considered for diagnosing patients' health conditions into specific grades, which can assist clinicians with proper treatment (Siontis et al. 2021). ECG measures the electricity of the heart, by analyzing each of electrical signal, it is possible to detect some abnormalities. In such conditions, ECG should allow continuous and remote monitoring.

Although the acquisition of ECG recordings is well standardized, human interpretations of ECG recordings vary widely. This is due to differences in the level of experience and expertise. To minimize these constraints, computer-generated interpretations have been used for various years. However, because this interpretation is based on predetermined rules and the limitations of the feature recognition algorithm, the interpretation results do not always capture the complexities and nuances contained in the ECG (Siontis et al. 2021). Based on this, the ECG by itself is often insufficient to diagnose several heart abnormalities. In myocardial infarction (MI), for example, is due to ST-segment deviation and may be occurred in other conditions such as acute pericarditis, left ventricular hypertrophy, left bundle-branch block, Brugada syndrome, and early repolarizations (Wang, Asinger, and Marriott 2003). Due of this, automatically diagnosing MI using a ruled based inference system from conventional ECG machine has a low reliability and by practice cardiologists are unable to diagnose it only from ECG record (Daly et al. 2012)(Cho et al. 2020). Furthermore, the traditional methods for diagnosing heart abnormalities specifically from a 12-lead ECG are difficult to apply in wearable devices (Walsh, Topol, and Steinhubl 2014) (Cho et al. 2020) and a wide variability in ECG morphology between patients causes major challenges.

The heart abnormalities analysis by using ECG signal processing can be conducted by using rhythm and beat feature. In the previous studies, such research has been proposed with several method. Deep learning (DL) is one type of artificial intelligence approach that can learn and extract meaningful patterns from complex raw data and recently has begun to widely used to analyze ECG signals for diagnosing an arrhythmia, heart failure, myocardial infarction, left ventricular hypertrophy, valvular heart disease, age, and sex with ECG alone and produce good result (Darmawahyuni et al. 2021) (Makimoto et al. 2020) (Attia et al. 2019) (Hannun et al. 2019) (Kwon et al. 2020(a)) (Kwon et al. 2020(b)) (Yildirim, 2018) (LeCun, Bengio, and Hinton 2015). DL performs excellently over a relatively short period of time. The sophistication of DL 
80 has a much better ability to feature representation at an abstract level compared to general 81 machine learning. The DL model can extract a hierarchical representation of the raw data 82 automatically and then utilize the last stacking layers to gain knowledge from complex features 83 to the simpler ones (Khan and Yairi 2018).

84 In the previous study, the ECG signal classification based on heart rhythm can be conducted with several features morphology of ECG signal like presenting ST-elevation and 86 depression, T-wave abnormalities, and pathological Q-waves (Ansari et al. 2017). Moreover, a 87 variety of ECG rhythm features, such as the R-R interval, S-T interval, P-R interval, and Q-T 88 interval have been implemented to automatically detect heart abnormalities over the past decade 89 (Gopika et al. 2020). Unlike an ECG rhythm, the efficiency classification of the irregular 90 heartbeat, either faster or slower than normal, or even waveform malformation can be improve 91 by using beat feature. (Khalaf, Owis, and Yassine 2015). For heartbeat classification, ECG pattern may be similar for different patients who have different heartbeats and may be different for the same patient at different times. ECG-based heartbeat classification is virtually a problem of temporal pattern recognition and classification (Zubair, Kim, and Yoon 2016)(Dong, Wang, and Si 2017). Based on the aforementioned instances, the variety of ECG signals with abnormalities must be handled specifically, either as an ECG rhythm or beat features.

Unfortunately, the challenge in analyzing the pattern of ECG signal is not limited to this. ECG signals have small amplitudes and short durations, measured in millivolts and milliseconds, respectively, and large inter- and intra-observer variability that influences the perceptibility of these signals (Lih et al. 2020). The analysis of thousands of ECG signals is time-consuming, and the possibility of misreading vital information is high. Automated diagnostic systems can utilize computerized recognition of heart abnormalities based on rhythm or beat to overcome such limitations. This could become the standard procedure by clinicians classifying ECG recordings.

Hence, the present study proposes a single DL architecture for classifying ECG patterns by using both rhythm and heartbeat features. Rather than treating ECG heartbeat and rhythm separately, we process both of them in the same framework. Hence, we only need a single DL architecture to classify the ECG signal with high accuracy. DL-based frameworks mainly include a stacked autoencoder (SAE), long short-term memory (LSTM), a deep belief network (DBN), convolutional neural networks (CNN), and so on. Among DL algorithms, we have generated a one-dimensional CNN (1D-CNN) model and showed promising results in our previous works (Nurmaini et al. 2020) (Tutuko et al. 2021). In other works, 1D-CNN has also performed well for ECG classification, with overall performances ranging from $93.53 \%$ to $97.4 \%$ accuracy using rhythm (Acharya et al. 2017); (Wang 2020) and with overall $92.7 \%$ to $96.4 \%$ accuracy using beat (Zubair et al. 2016) (Kiranyaz, Ince, and Gabbouj 2015). For the pattern recognition technique, $1 \mathrm{D}-\mathrm{CNN}$ is well known, as it integrates feature extraction, dimensionality reduction, and classification techniques utilizing several convolution layers, pooling layers, and a fully connected layer. Convoluted optimum features are derived and classified using feed-forward artificial neural networks using a fully connected layer with a learning framework for back propagation ( $\mathrm{Li}$ et al. 2019). This study and the proposed approach make the following novel 
120

121

122

123

124

125

126

127

128

129

130

131

132

133

134

135

136

137

138

139

140

141

142

143

144

145

146

147

148

149

150

151

152

153

154

155

contributions:

- Proposes the generalization framework of deep learning for ECG signal classification with high accuracy in intra and inter-patients' scenario;

- Develops a single DL-architecture for classifying ECG signal pattern based on both of rhythm and beat feature with simple segmentation;

- Validates the proposed framework with five public ECG datasets that have different frequency sampling with massive data; and

- Experiment with 24-class abnormalities found in the ECG signal, consisting of nine-class of ECG pattern based on rhythms feature and 15-class of ECG pattern based on beats feature.

The rest of this paper is organized as follows. Section 2 presents the materials and methods, which comprise ECG raw data and the proposed methodology for ECG rhythm and beat classification using 1D-CNN architecture. Section 3 presents the results and discussion. Finally, the conclusions are presented in Section 4.

\section{Materials and Methods}

\section{Data Preparation}

In this study, we use the public data set from PhysioNet (Goldberger et al. 2000). The ECGs data in the Physionet were collected from healthy volunteers and patients with different heart diseases. This database has already been published online by a third party and unrelated to the study. Consequently, there should be no concerns regarding the ethical disclosure of the information. To process the ECG signal pattern recognition, we utilize two segmentation processes, rhythm and beat. Therefore the experimental databases is divided into two cases, (i) for ECG rhythm classification utilize the PTB Diagnostic ECG (PTB DB) (Bousseljot, Kreiseler, and Schnabel 1995), the BIDMC Congestive Heart Failure (CHF) (Baim et al. 1986), the China Physiological Signal Challenge 2018 (Liu et al. 2018), the MIT-BIH Normal Sinus Rhythm (Goldberger et al. 2000); and (ii) for ECG beat classification utilize the MIT-BIH Arrhythmia Database (Moody and Mark 2001).

A summary of each database is provided as follows:

- The PTB DB contains 549 records from 290 patients (209 men and 81 women). ECG signals were sampled at $1000 \mathrm{~Hz}$. Each ECG record includes 15 signals measured simultaneously: 12 conventional leads (I, II, III, aVR, aVL, aVF, V1, V2, V3, V4, V5, V6) along with three ECG Frank leads (vx, vy, vz) in the .xyz file. For this study, only a single lead (lead II) was used. The database provides ECG normal and nine heart abnormalities, such as myocardial infarction, cardiomyopathy, bundle branch block, dysrhythmia, hypertrophy, myocarditis, and valvular heart disease. 
156

157

158

159

160

161

162

163

164

165

166

167

168

169

170

171

172

173

174

175

176

177

178

179

180

181

182

183

184

185

186

187

188

189

190

191

192

- The BIDMC CHF database includes ECG recordings of about 20 hours in duration from 15 patients with severe congestive heart failure conditions, that is, New York Heart Association (NYHA) Class 3 and 4. The database contains two ECG signals that are sampled at 250 samples per second.

- The China Physiological Signal Challenge 2018 database was collected from 11 hospitals and contains 12-lead ECG recordings lasting 6-60 seconds. All recordings were sampled at $500 \mathrm{~Hz}$. The database provides three training sets, and all training sets were used in this study. However, only lead II was used for this study.

- The MIT-BIH Normal Sinus Rhythm includes 18 long-term ECG recordings that were found to have no significant arrhythmias. The database contains five male and 13 female patients. ECG recordings were sampled at $128 \mathrm{~Hz}$.

- The MIT-BIH Arrhythmia Database use for beat classification. This study experimented with which was obtained from 47 different patients. It contained 48 half-hour excerpts from two-channel ambulatory ECG recordings. All ECG recordings were digitized at 360 samples per second. For the present study, we utilized ECG beat types of entire records. Most of the beats recorded in these databases have annotations associated with the type of beat or the events.

The total records, labels, and plots of the nine-class pattern for ECG rhythm classification are presented in Table 1 and Figure 1, and the 15-class pattern for ECG beat classification were analyzed for this study are presented in Table 2 and Figure 2.

\section{Proposed Methodology of 1D-CNN}

In this study, we present a methodology using single DL architecture to classify 25 classes of ECG signal pattern of based on rhythm and beat feature. Unlike others methodologies that treat beat and rhythm separately, our approach enables both forms to proceed on a single DL architecture. The methodology includes ECG signal denoising, beat and rhythm segmentation and classification. Using this approach, pattern abnormalities that occurred in the ECG, both in beats and rhythms, can be detected only using single architecture. We generalized the 1D-CNN architecture that was published in previous work (Nurmaini et al. 2020) (Tutuko et al. 2021). The proposed methodology of the 1D-CNN generalized architecture is presented in Figure 3 . The general process in the methodology with standardize the evaluation process considering a clinical point of view. This standardization and defined the workflow to perform the evaluation to make sure the experiments are reproducible and comparable. Aiming to standardize, the general methodology including five main stages are, (i) data base selection from the ECG public database; (i) the pre-processing stage of ECG signals by eliminating various kinds of noise and artifacts using discrete wavelet transforms; (iii) the segmentation stage for ECG signal based on rhythm and beat. The ECG signal segmented to 2700 and 252 nodes, respectively; (iv) 1D-CNN, as the feature extraction and classifier, learn the characteristics of each rhythm and beat episodes 
193 for ECG signal classification. and (v) the evaluation stage of the proposed model based on

194 validation and testing data with accuracy, sensitivity, specificity, precision and F1-score.

195

196

197

198

199

200

201

202

203

204

205

206

207

208

209

210

211

212

213

214

215

216

217

218

219

220

221

222

223

224

225

226

227

228

229

230

\section{Database Selection}

We have total of 168,472 rhythm episodes and 110,082 beat episodes as ECG features were used for training, validation, and an testing (as unseen data). The 1D-CNN architecture was used to classify the nine-class by using rhythms feature segmentation and 15-class of beats feature segmentation of the ECG signal. The information available from the single-lead ECG standard recordings included different signal lengths and frequency samplings $(128,250,500$, and $1000 \mathrm{~Hz})$.

\section{Pre-processing}

The ECG signal can become corrupted during acquisition due to different types of artifacts and interference, such as muscle contraction, baseline drift, electrode contact noise, and power line interference (Sameni et al. 2007)(Tracey and Miller 2012)(Wang et al. 2015). To achieve an accurate analysis and diagnosis, undesirable noise and signals should be removed or deleted from the ECG by eliminating various kinds of noise and artifacts. This study implemented DWT as a frequently used denoising technique that offers a useful option for denoising ECG signals. This study also implemented some wavelet families for ECG signals, such as symlets, daubechies, haar, bior, and coiflet, to analyze which type of wavelet would obtain the best signal denoising result. Among them, based on the highest the signal noise to ratio (SNR) results, the symlet wavelet was the best DWT parameter and was chosen for ECG signal denoising.

\section{ECG Signal Segmentation}

The aim of ECG segmentation is to divide a signal into many parts with similar statistical properties, such as amplitude, nodes, and frequency. The presence, time, and length of each segment of an ECG signal have diagnostic and biophysical significance, and the various sections of an ECG signal have distinctive physiological meaning (Yadav and Ray 2016). ECG signal segmentation may also be accurately analyzed. The process of ECG feature segmentation for rhythm and beat classification can be described as follows:

- ECG rhythm segmentation is the process to produce the features for the entire ECG signal recordings at 2700 nodes without considering the different frequency sampling $(128,250,500$, and $1000 \mathrm{~Hz})$ for ECG rhythm classification. In our previous work (Nurmaini et al. 2020), we successfully segmented the length of AF episodes to 2700 nodes. Therefore, for this study, we generated the features for nine-class of normalabnormal ECG rhythm. The length of 2700 nodes contained at least two R-R intervals between one and the next beat with different frequency samplings in all records. Furthermore, the 2700-node segmentations might show more than two R-R intervals with a minimum frequency sampling of $128 \mathrm{~Hz}$ for the training, validation, and unseen 
231

232

233

234

235

236

237

238

239

240

241

242

243

244

245

246

247

248

249

250

251

252

253

254

255

256

257

258

259

260

261

262

263

264

265

266

267

set. As a result, the best ECG episodes were chosen from 2700 nodes for segmentation. The process of ECG rhythm classification is illustrated in Figure 4(a). Figure 4(a) shows that all lengths of the ECG recordings have been segmented to each episode of 2700 nodes. If the total nodes were less than 2700 nodes, we added the zero-padding technique, which involved extending a signal with zeros.

- ECG beat segmentation is the step of intercepting numerous nodes in a signal to discern not only subsequent heart beats but also the waveforms included in each beat (Qin et al. 2017). The former refers to the characteristics retrieved from a single beat, which typically only contains one R-peak. The latter, however, refers to features that are dependent on at least two beats. These features include more information than a single R-peak. The waveforms of beat segmentation are presented in Figure 4(b). Figure 4(b) shows the positions of the P-wave, QRS-complex, and T-wave, which are all intimately connected to the location of the R-peak. According to (Qin et al. 2017)(Chang et al. 2012)(Nurmaini et al. 2019), the average ECG rhythm frequency is between 60 and 80 beats per minute, the t 1 duration is 0.25 seconds before R-peak, and the $\mathrm{t} 2$ duration is 0.45 seconds after R-peak, which results in a total length of 0.7 seconds. A total of 0.7 seconds contains 252 nodes, with a sampling frequency of 360 $\mathrm{Hz}$, which covers the P-wave, QRS-complex, and T-wave (one beat).

\section{Feature Extraction and Classification}

The 1D-CNN classifier was proposed by (Nurmaini et al. 2020) for AF detection. By using the architecture, we generalized the model for abnormal-normal rhythm and beat classification. The rectified linear unit (ReLU) function was adopted with 13 convolution layers $(64,128,256$, and 512 filters) and also consisted of five max pooling layers. The 1D-CNN model comprised two fully connected layers with 1000 nodes for each layer and one node for the output layer. The 1D-CNN required a three-dimensional input, which consists of $n$ samples, $n$ features, and timesteps. The detailed process of 1D-CNN architecture for both ECG rhythm and beat classification was as follows:

- For ECG based on rhythm classification, the input timesteps with the dimension $2700 \times 1$ were fed into the convolution layer equipped with the ReLU activation function. The first and second convolutional layers produced an output length of 64 with a kernel size of 3 . The output of the first and second convolutional layers through the max pooling layer had a kernel size of 2 for the feature reduction. The output of the first max pooling layer as the input for the third and fourth convolutional layers produced 128 feature maps. The convolutional layers were passed onto the fifth and last convolutional layers and produced output lengths of 256 and 512, respectively, with a kernel size of 3 . The output of the last convolutional layer was then passed onto two fully connected layers with a total of 
268

269

270

271

272

273

274

275

276

277

278

279

280

281

282

283

284

285

286

287

288

289

290

291

292

293

294

295

296

297

298

299

300

301

302

303

304

305

306

1000 nodes. This architecture produced an output of a nine-class ECG rhythm classification.

- Unlike ECG rhythm classification, none of the processes differed from the features interpretation for ECG based on beat classification. The main differences were the input timesteps value of $(252,1)$ and products of the output size of the 15-class ECG beat classification. The architecture also implemented the ReLU activation function with 64, 128, 256, and 512 filters, with a kernel size of 3. For each max pooling layer, a kernel size of 2 was also used for the feature interpretation of the 1D-CNN for ECG beat classification.

\section{Model Evaluation}

Classification ECG signal based on rhythm and beat feature is evaluated by using intra and inter patient scheme. Such schemes are conducted to resemble a clinical environment and to ensure the robustness of the proposed model. Five commons metrics used in this study are accuracy, sensitivity, specificity, precision and F1-score. Moreover, two measures are usually considered for evaluating the classification performance, specifically for imbalance data, are receiver-operating characteristic (ROC) and Precision-Recall (P-R) curves. These two-evaluation metrics were added because the overall accuracy was distorted by the majority class results, since the beat type classes are extremely imbalanced in the available dataset.

\section{Results and Discussion}

For ECG rhythm classification, the proposed 1D-CNN model was tested on an unseen set, but ECG beat classification was only tested on the validation set in this study. All experimentation in the training processes used a 10-fold cross-validation scheme. This scheme divides the collection of observations into $k$ groups, or folds, of roughly similar size at random. The scheme is fitted on the remaining $k-1$ folds, with the initial fold serving as a validation set. For the selected model, the parameters that provided the best cross-validation accuracy, sensitivity, specificity, precision, and F1 score were chosen.

\section{ECG Rhythm Classification in Validation Model}

A total of 2445 records consisted of rhythm episodes of 138,415 training sets, 15,373 validation sets, and 14,684 unseen sets after being segmented by each 2700 nodes (refer to Table 3). A total of 168,472 episodes were analyzed for the ECG rhythm classification task. As can be seen, all PTB Diagnostics ECG records were used for the training and validation sets. The rest of the datasets were used for the training, validation, and unseen sets. Table 3 shows the large different ratio between one class and another (imbalanced) class, for example, a total number of MI, HF, and HC classes to H, M, and VHD classes. However, we did not implement the oversampling techniques to overcome such a case in this study. 
307

308

309

310

311

312

313

314

315

316

317

318

319

320

321

322

323

324

325

326

327

328

329

330

331

332

333

334

335

336

337

338

339

340

341

342

343

344

345

346

Without considering the data ratio (total number) of episodes for each class, we validated the proposed 1D-CNN model to the 10-fold cross-validation scheme. Figure 5 shows the performance results of folds 1 through 10, which were evaluated for accuracy, sensitivity, specificity, precision, and F1 score. The performance results obtained above $99 \%$ for accuracy and specificity and ranged from 93 to $99 \%$ for sensitivity, precision, and F1 score. The model with the highest accuracy was chosen as the best model for this study out of all the models analyzed. The model had an accuracy of $99.98 \%$, a sensitivity of $98.53 \%$, a specificity of $99.99 \%$, a precision of $99.81 \%$, and an F1 score of $99.15 \%$ (fold 6 ). The results showed that the proposed 1D-CNN was the most accurate predictor, with an accuracy of $99.98 \%$.

Confusion Matrix (CM) for rhythm evaluation result is shown in Figure 6. This metric is used to capture information about the predicted results from the model respected to the actual label. It can be seen that the BBB class has four prediction errors (predicted as healthy-control class) and two healthy-control rhythms that are predicted as BBB. The prediction error between those arises due to the morphology of these types of rhythms is almost similar. Even so, the overall predictive results of the proposed approach provide a satisfactory evaluation performance. Based on the CM, the classification result of the ECG signal with rhythm feature produces good performance, due to only two class of ECG pattern have misclassified (HC and HF). However, overall result can be state that the classification is close to $100 \%$.

Using the performance value in $\mathrm{CM}$, we can observe the classification result with other views in terms of classification model at all classification thresholds named receiver-operating characteristic (ROC) and precision-recall (PR) curves. This curve plots two parameters true positive rate (sensitivity) and false positive rate (specificity) provide a graphical representation of a classifier's performance across many thresholds, rather than a single value. It is important to understand the trade-off in performance for different threshold values. As shown in Figures 7(a) and 7(b). Figure 7(a) shows the resulting ROC curve, which compares the nine-class of ECG rhythm characteristics. The comparable value is sensitivity versus specificity. Sensitivity is the ability to correctly identify the true positive class of ECG rhythm, whereas specificity is the ability to correctly identify the true negative rate of ECG rhythm. Therefore, if used in medical data, it will produce a precise and accurate diagnosis. Misclassification between positive class and negative class of ECG rhythm can be dangerous, and the consequences can be as serious as death.

The area under the curve (AUC) is the value analyzed in the ROC by looking at how far the middle value is and whether the area below the curve approaches the value of 1 . The lower left point of the graph $(0,0)$ is a value that does not contain errors (no false positives) and does not detect any true positives. On the upper right side of the graph $(1,1)$, the opposite point defines all true positives but with a $100 \%$ error rate (rates of false positives). The upper left point $(0,1)$ is the ideal classification that defines all true positives without any mistakes (no false positives or 0 cost). The lower right point $(1,0)$ is the worst classification, where all subjects labeled as positive are simply false positives, without knowing true positives. As shown in Figure 7(a), the ROCs of the nine-class normal-abnormal ECG rhythm show excellent performance, as the value of the 
347 ROC for the nine-class classification is 1 , or the AUC is about $100 \%$. This means that the

348 proposed 1D-CNN can categorize all classes with higher accuracy and precision. However, the

349

350

351

352

353

354

355

356

357

358

359

360

361

362

363

364

365

366

367

368

369

370

371

372

373

374

375

376

377

378

379

380

381

382

383

384

385

386 ROC cannot be trusted with imbalanced data, and it remains unchanged even after the performance changes. Therefore, the P-R curve is used to describe the classifier performance on imbalanced data (Figure 7(b)). The overall performances are also good, as the P-R value is 1 .

Table 4 lists the performance results for the nine-class of ECG based on rhythm feature. As can be seen, the C, D, H, MI, M, and VHD classes obtained 100\% for accuracy, sensitivity, specificity, precision, and F1 score. The proposed 1D-CNN model was proven to be robust and had no effect on the imbalanced class problem. For the nine-class classification, the average of all performance metrics achieved above $99 \%$ accuracy.

\section{ECG Beat Classification in Validation Model}

For the 15-class of ECG beats, a total of 110,082 beats were trained, validated, and tested (unseen) in this study. The large different ratio between one class and another (imbalanced) class, however in this study we can't conducted the augmentation data. All ECG beats data divided into a ratio of $8: 2$ or $80 \%$ is used for training data and the remaining for testing. The process of training with 10 -fold is selected with randomly. Therefore, about 88,065 beats are used as training data and about 22,017 beats as testing data.

The performance results of folds 1 through 10 using the 10-fold cross-validation scheme are shown in Figure 8. As can be seen, the results vary from $0 \%$ as the lowest and around $99 \%$ as the highest result. Accuracy and specificity achieved above $99 \%$, sensitivity and precision ranged from above $0 \%$ to $94 \%$, and the $\mathrm{F} 1$ score ranged from $0 \%$ to $94 \%$. The model had an accuracy of $99.88 \%$, a sensitivity of $96.98 \%$, a specificity of $99.90 \%$, a precision of $92.24 \%$, and an F1 score of $94.39 \%$ (fold 6). Unlike the ECG rhythm results, the performance of the 10-fold was not good enough. There was an outlier of sensitivity, which had a 0 (zero) value in the initial fold. The massive difference between the total number of the normal beat $(\mathrm{N})$ class and the other abnormal beats could be an imbalanced class problem.

To analyze the performance of the 15-class of ECG beats, we also presented the confusion matrix evaluation in Figure 9. It can be seen that normal beats have the highest number of true positives (with 7248 data). However, this beat also has the most false-negative and false positive values with 46 and 21 data, respectively compared to other classes. Furthermore, both classes $\mathrm{J}$ and e have neither false positive nor false negative errors. Even though the ratio of number data used in this study was imbalance, the atrial escape beat (e), which is proven to be a minority class, was able to classify by the model correctly. However, an imbalanced data problem still requires a particular concern to avoid the model simply predicting the majority class rather than the minority.

To analyze the performance of the 15-class of ECG beats, we also presented the ROC and P-R curve (refer to Figures 9(a) and 9(b)). Figure 9(a) shows that the perfect classification can be presented in the R, L, j, and P beat classes. However, the other beat classes obtained an AUC value above $75 \%$. Also, Figure 9(b) shows the worst classification as the e beat class, with an 
387 AUC value above $50 \%$. According to the ratio of number data that was used in this study, the 388 atrial escape beat (e) is proven to be a minority class, as it has limited dataset representation. Due 389 to the large imbalanced class, the model tends to perform poorly and requires some 390 modifications to avoid simply predicting the majority class in all cases.

391

392

393

394

395

396

397

398

399

400

401

402

403

404

405

406

407

408

409

410

411

412

413

414

415

416

417

418

419

420

421

422

423

424

425

426

The results for the 15-class ECG beat classification are listed in Table 5. As can be seen, the results show an above $99 \%$ accuracy and specificity for all 15-class of ECG beats. The results presentation is quite good for the ECG beat classification task, although some beats' results (A, a, and $\mathrm{j}$ ) are not. The $\mathrm{A}$ and a beats are related to an atrial premature beat, causing aberrant ventricular conduction. An unexpected beat discharged by an ectopic focus in the atria is termed a premature atrial beat. While some fibers are still refractory, the impulse from the premature beat reaches the His-Purkinje system early. Due to abnormal ventricular conduction, the resultant QRS complex exhibits a right BBB pattern. Also, the $\mathrm{j}$ beat is a delayed heartbeat originating from an ectopic focus in the atrioventricular junction. The classification of ECG beats tends to be more challenging because the results are related to the heart beat segmentation process, which will be close to optimal with the QRS detection.

\section{ECG signal Classification with Inter-patient Data}

Tables 4 and 5 list the proposed model result with dataset based on intra-patient scenario. Such conditions where the ECG data from the same patients probably appear in the training and validation set. In this study, we took the precaution to construct and evaluate the classification using rhythm and beat features also from different patients (inter-patient). To test the robustness of the proposed 1D-CNN model, we tested the model on an unseen set (refer to Table 6). The unseen set sample consisted of five of the 24-class of ECG-based rhythms and beat featureBBB, HC, HF, V and L class. From the experiment, the performance still achieved outstanding results.

\section{Benchmarking of The Proposed Model}

The comparison results of our proposed 1D-CNN architecture with the state-of-the-art model are listed in Table 7. In order to make a fair benchmarking, we compare our proposed model with several previous studies. These studies focus on the ECG signal classification using DL architecture, especially the use of 1D-CNN architecture (Yildirim et al. 2018) (Rajkumar et al. 2019) (Nannavecchia et al. 2021), LSTM architecture (Yildirim et al. 2019) (Gao et al. 2019), and combination architecture of 1D-CNN as a feature extraction and LSTM as a classifier (Lui, and Chow 2018) (Oh et al. 2018) (Yildirim et al. 2020) (Chen et al. 2020) (Luo et al. 2021). However, all the classification methodologies are developed by treating beat and rhythm separately. In contrast, our study utilizes a single architecture based on 1D-CNN architecture through both features, rhythm and beat, to classify 24 patterns of ECG signals. In the ECG signal interpretation, the abnormalities can be analyzed using heart beat or heart rhythm feature. Therefore, such process is more efficient to integrate two features for classifying ECG signals in one architecture. To our 
427 knowledge, no studies developed such combination scenario with one architecture. Thus, in this

428

429

430

431

432

433

434

435

436

437

438

439

440

441

442

443

444

445

446

447

448

449

450

451

452

453

454

455

456

457

458

459

460

461

462

463

464

465

466 study, we will analyze and compare the classification results for beat and rhythm features separately.

It can be seen in Table 7, the previous studies show that the ECG signal classification based on rhythm feature propose 1D-CNN architecture for 17 classes with $91.30 \%$ accuracy (Yildirim et al. 2018), and propose LSTM architecture for five classes with 99.23\% accuracy (Yildirim et al. 2019). From these two studies, the classification performance is improved; however, the number of classes are reduced from 17 to 5-class. Other study for ECG signal classification based on beat feature utilize combination between 1D convolutional layers and LSTM architecture with 10.000 subject and seven-class abnormalities (Yildirim et al. 2020). By using the proposed model, the classification accuracy around $92.24 \%$, unfortunately, the sensitivity only reaches $80.15 \%$. It means that the smallest change in the ECG signal can't be detected by the network. They use convolutional layers to produce a low- and high-level feature, however, the LSTM classifiers lack to recognize the dynamic of ECG feature extracted from CNN. The sensitivity is important value in medical analysis, its relation to the number of false-negative result. The small sensitivity value indicates that many ECG signals are misclassified. Such case also occurred in (Lui and Chow 2018), they use 1D-CNNLSTM architecture, however the sensitivity only reaches $92.40 \%$.

Combination of 1D Convolutional layers with other DL architecture can actually produce quite impressive results (Lui, and Chow 2018) (Oh et al. 2018) (Chen et al. 2020) (Yildirim et al. 2020) (Luo et al. 2021) rivalling a model which uses individual 1D-CNN and LSTM architecture (Yildirim et al. 2018) (Gao et al. 2019) (Rajkumar et al. 2019) (Nannavecchia et al. 2021). Even in other study, in order to obtain satisfactory results in ECG signal classification, three different DL architecture, 1D-CNN, LSTM and GRU are combined (Luo et al. 2021). Unfortunately, they used SMOTE algorithm to eliminate the imbalanced problems. By using resampling methods can increase the overlapping between classes and can introduce additional noise. The beat classification is still challenging, when the number of classes is increased it will decline the performance. In (Nannavecchia et al. 2021), they classify ECG signal for 21 classes abnormalities. However, the classification result is unsatisfactory with $89.51 \%$ accuracy and $87.79 \%$ sensitivity, which means a large of number classes are misclassified.

In our proposed model only utilize 1D-CNN architecture with 13 convolutional layers and five max-pooling layers, we produce a satisfactory result using both beat and rhythm features. Our model performance outperforms other studies with a large number of classes. All the performance of nine-class classification value reach over $99 \%$ by using beats feature, but the classification sensitivity decreases to $96.67 \%$ by using rhythm feature. It happened because we use 15 classes of ECG signal abnormalities with an unbalanced number of classes. Such condition causes the classifiers tend to make biased learning model that has a poorer predictive accuracy over the minority classes compared to the majority classes. Even though, our model still maintains the classification performance with imbalanced data, the sensitivity value is decreased only $3 \%$, it does not affect the performance significantly. One dimensional convolutional learning methods are more efficient to learn local patterns than a recurrent neural network. It contains a sliding filter,

Peer] Comput. Sci. reviewing PDF | (CS-2021:07:64082:2:0:NEW 22 Nov 2021) 
467

468

469

470

471

472

473

474

475

476

477

478

479

480

481

482

483

484

485

486

487

488

489

490

491

492

493

494

495

496

497

498

499

500

\section{1}

502

503

504

505

which may be regarded as moving across the input by sharing weights over a local patch function. It concludes that 1D-CNN performs better in areas where local patterns are important for classification task.

Although the results look promising for ECG rhythm and beat classification, there are some limitations to our study:

- The pre-processing stage of the ECG signal still needs improvement, specifically in the case of ECG signals that have different sampling frequencies, leads, and various noises.;

- The segmentation of the P, QRS, and T-waves and the HRV measurement before the classification process were not carried out; and

- The proposed model was not validated against the hospital patient data. We only used the available public dataset.

\section{Conclusions}

Deep learning has gained a central position in recent years for ECG rhythm and beat classification. It was built on a foundation of significant algorithmic details and generally can be understood in the construction and training of DL architectures. A DL approach based on one 1D-CNN architecture has been presented to automatically learn and classify the nine-class of ECG pattern with rhythms feature and 15-class of ECG pattern with beats feature, which is important for classifying the abnormalities pattern. In this study, the proposed 1D-CNN model, which consisted of 13 convolutional layers and five max-pooling layers, was used. The 1D-CNN has low computational requirements. Thus, it is well-suited for real-time and low-cost applications for ECG devices.

Using the 10-fold cross-validation scheme, the performance results had an accuracy of $99.98 \%$, a sensitivity of $99.90 \%$, a specificity of $99.89 \%$, a precision of $99.90 \%$, and an F1 score of $99.99 \%$ for ECG rhythm classification. Also, for ECG beat classification, the model obtained an accuracy of $99.87 \%$, a sensitivity of $96.97 \%$, a specificity of $99.89 \%$, a precision of $92.23 \%$, and an F1 score of $94.39 \%$. We realize the performance results of the ECG rhythm are better than the ECG beat classification. The selection of an appropriate preprocessing step for QRS detection to accurately find the R-peak to achieve the best model for ECG beat classification is needed to achieve high performance results. In the future, the challenges regarding ECG signals are still many, such as the precision segmentation of P, QRS, and T-waves before the process of rhythm and beat classification.

\section{Acknowledgements}

This work was supported by Intelligent System Research Group (IsysRG), Universitas Sriwijaya, Indonesia.

\section{References}


506

507

508

509

510

511

512

513

514

515

516

517

518

519

520

521

522

523

524

525

526

527

528

529

530

531

532

533

534

535

536

537

538

539

540

541

542

543

544

545
Acharya, U. Rajendra, Hamido Fujita, Shu Lih Oh, Yuki Hagiwara, Jen Hong Tan, and Muhammad Adam. 2017. "Application of Deep Convolutional Neural Network for Automated Detection of Myocardial Infarction Using ECG Signals." Information Sciences 415:190-98.

Ansari, Sardar, Negar Farzaneh, Marlena Duda, Kelsey Horan, Hedvig B. Andersson, Zachary D. Goldberger, Brahmajee K. Nallamothu, and Kayvan Najarian. 2017. "A Review of Automated Methods for Detection of Myocardial Ischemia and Infarction Using Electrocardiogram and Electronic Health Records." IEEE Reviews in Biomedical Engineering 10:264-98.

Attia, Zachi I., Suraj Kapa, Francisco Lopez-Jimenez, Paul M. McKie, Dorothy J. Ladewig, Gaurav Satam, Patricia A. Pellikka, Maurice Enriquez-Sarano, Peter A. Noseworthy, Thomas M. Munger, Samuel J. Asirvatham, Christopher G. Scott, Rickey E. Carter, and Paul A. Friedman. 2019. "Screening for Cardiac Contractile Dysfunction Using an Artificial Intelligence-Enabled Electrocardiogram." Nature Medicine 25(1):70-74.

Baim, Donald S., Wilson S. Colucci, E. Scott Monrad, Harton S. Smith, Richard F. Wright, Alyce Lanoue, Diane F. Gauthier, Bernard J. Ransil, William Grossman, and Eugene Braunwald. 1986. "Survival of Patients with Severe Congestive Heart Failure Treated with Oral Milrinone." Journal of the American College of Cardiology 7(3):661-70.

Bousseljot, R., D. Kreiseler, and A. Schnabel. 1995. "Nutzung Der EKG-Signaldatenbank CARDIODAT Der PTB Über Das Internet.” Biomedizinische Technik/Biomedical Engineering 40(s1):317-18.

Chang, Pei-Chann, Jyun-Jie Lin, Jui-Chien Hsieh, and Julia Weng. 2012. "Myocardial Infarction Classification with Multi-Lead ECG Using Hidden Markov Models and Gaussian Mixture Models." Applied Soft Computing 12(10):3165-75.

Chen, Chen, Zhengchun Hua, Ruiqi Zhang, Guangyuan Liu, and Wanhui Wen. 2020. "Automated Arrhythmia Classification Based on a Combination Network of CNN and LSTM.” Biomedical Signal Processing and Control 57:101819.

Cho, Younghoon, Joon-myoung Kwon, Kyung-Hee Kim, Jose R. Medina-Inojosa, Ki-Hyun Jeon, Soohyun Cho, Soo Youn Lee, Jinsik Park, and Byung-Hee Oh. 2020. "Artificial Intelligence Algorithm for Detecting Myocardial Infarction Using Six-Lead Electrocardiography." Scientific Reports 10(1):20495.

Daly, MJ, DD Finlay, D. Guldenring, CD Nugent, A. Tomlin, B. Smith, AAJ Adgey, and MT Harbinson. 2012. "Detection of Acute Coronary Occlusion in Patients with Acute Coronary Syndromes Presenting with Isolated ST-Segment Depression.” European Heart Journal: Acute Cardiovascular Care 1(2):128-35.

Darmawahyuni, Annisa, Siti Nurmaini, Muhammad Naufal Rachmatullah, Firdaus Firdaus, and Bambang Tutuko. 2021. "Unidirectional-Bidirectional Recurrent Networks for Cardiac Disorders Classification." Telkomnika 19(3):902-10.

Dong, Xunde, Cong Wang, and Wenjie Si. 2017. "ECG Beat Classification via Deterministic Learning." Neurocomputing 240:1-12.

Peer] Comput. Sci. reviewing PDF | (CS-2021:07:64082:2:0:NEW 22 Nov 2021) 
546 Gao, Junli, Hongpo Zhang, Peng Lu, and Zongmin Wang. 2019. "An Effective LSTM Recurrent

547

548

549

550

551

552

553

554

555

556

557

558

559

560

561

562

563

564

565

566

567

568

569

570

571

572

573

574

575

576

577

578

579

580

581

582

583

584

585

Network to Detect Arrhythmia on Imbalanced ECG Dataset." Journal of Healthcare Engineering 2019.

Goldberger, Ary L., Luis A. N. Amaral, Leon Glass, Jeffrey M. Hausdorff, Plamen Ch Ivanov, Roger G. Mark, Joseph E. Mietus, George B. Moody, Chung-Kang Peng, and H. Eugene Stanley. 2000. "PhysioBank, PhysioToolkit, and PhysioNet: Components of a New Research Resource for Complex Physiologic Signals." Circulation 101(23):e215--e220.

Hannun, Awni Y., Pranav Rajpurkar, Masoumeh Haghpanahi, Geoffrey H. Tison, Codie Bourn, Mintu P. Turakhia, and Andrew Y. Ng. 2019. "Cardiologist-Level Arrhythmia Detection and Classification in Ambulatory Electrocardiograms Using a Deep Neural Network." Nature Medicine 25(1):65-69.

Khalaf, Aya F., Mohamed I. Owis, and Inas A. Yassine. 2015. "A Novel Technique for Cardiac Arrhythmia Classification Using Spectral Correlation and Support Vector Machines." Expert Systems with Applications 42(21):8361-68.

Khan, Samir and Takehisa Yairi. 2018. "A Review on the Application of Deep Learning in System Health Management." Mechanical Systems and Signal Processing 107:241-65.

Kiranyaz, Serkan, Onur Avci, Osama Abdeljaber, Turker Ince, Moncef Gabbouj, and Daniel J. Inman. 2021. "1D Convolutional Neural Networks and Applications: A Survey." Mechanical Systems and Signal Processing 151:107398.

Kiranyaz, Serkan, Turker Ince, and Moncef Gabbouj. 2015. "Real-Time Patient-Specific ECG Classification by 1-D Convolutional Neural Networks." IEEE Transactions on Biomedical Engineering 63(3):664-75.

Kwon, Joon-Myoung, Ki-Hyun Jeon, Hyue Mee Kim, Min Jeong Kim, Sung Min Lim, KyungHee Kim, Pil Sang Song, Jinsik Park, Rak Kyeong Choi, and Byung-Hee Oh. 2020(a). "Comparing the Performance of Artificial Intelligence and Conventional Diagnosis Criteria for Detecting Left Ventricular Hypertrophy Using Electrocardiography." EP Europace 22(3):412-19.

Kwon, Joon-Myoung, Soo Youn Lee, Ki-Hyun Jeon, Yeha Lee, Kyung-Hee Kim, Jinsik Park, Byung-Hee Oh, and Myong-Mook Lee. 2020(b). "Deep Learning-Based Algorithm for Detecting Aortic Stenosis Using Electrocardiography." Journal of the American Heart Association 9(7).

LeCun, Yann, Yoshua Bengio, and Geoffrey Hinton. 2015. "Deep Learning." Nature 521(7553):436-44.

Li, Zhangjun, Xujian Feng, Ziqian Wu, Cuiwei Yang, Baodan Bai, and Qunqing Yang. 2019. "Classification of Atrial Fibrillation Recurrence Based on a Convolution Neural Network with SVM Architecture." IEEE Access 7:77849-56.

Lih, Oh Shu, V. Jahmunah, Tan Ru San, Edward J. Ciaccio, Toshitaka Yamakawa, Masayuki Tanabe, Makiko Kobayashi, Oliver Faust, and U. Rajendra Acharya. 2020. "Comprehensive Electrocardiographic Diagnosis Based on Deep Learning." Artificial Intelligence in Medicine 103:101789.

Peer] Comput. Sci. reviewing PDF | (CS-2021:07:64082:2:0:NEW 22 Nov 2021) 
586

587

588

589

590

591

592

593

594

595

596

597

598

599

600

601

602

603

604

605

606

607

608

609

610

611

612

613

614

615

616

617

618

619

620

621

622

623

624

625

626

Liu, Feifei, Chengyu Liu, Lina Zhao, Xiangyu Zhang, Xiaoling Wu, Xiaoyan Xu, Yulin Liu, Caiyun Ma, Shoushui Wei, Zhiqiang He, and others. 2018. "An Open Access Database for Evaluating the Algorithms of Electrocardiogram Rhythm and Morphology Abnormality Detection." Journal of Medical Imaging and Health Informatics 8(7):1368-73.

Lui, Hin Wai and King Lau Chow. 2018. "Multiclass Classification of Myocardial Infarction with Convolutional and Recurrent Neural Networks for Portable ECG Devices." Informatics in Medicine Unlocked 13:26-33.

Luo, Xinyu, Liuyang Yang, Hongyu Cai, Rui Tang, Yu Chen, and Wei Li. 2021. "MultiClassification of Arrhythmias Using a HCRNet on Imbalanced ECG Datasets." Computer Methods and Programs in Biomedicine 208:106258.

Makimoto, Hisaki, Moritz Höckmann, Tina Lin, David Glöckner, Shqipe Gerguri, Lukas Clasen, Jan Schmidt, Athena Assadi-Schmidt, Alexandru Bejinariu, Patrick Müller, Stephan Angendohr, Mehran Babady, Christoph Brinkmeyer, Asuka Makimoto, and Malte Kelm. 2020. "Performance of a Convolutional Neural Network Derived from an ECG Database in Recognizing Myocardial Infarction." Scientific Reports 10(1).

Moody, George B. and Roger G. Mark. 2001. "The Impact of the MIT-BIH Arrhythmia Database." IEEE Engineering in Medicine and Biology Magazine 20(3):45-50.

Nannavecchia, Antonella, Francesco Girardi, Pio Raffaele Fina, Michele Scalera, and Giovanni Dimauro. (2021). "Personal Heart Health Monitoring Based on 1D Convolutional Neural Network." Journal of Imaging 7(2): 26.

Nurmaini, Siti, Alexander Edo Tondas, Annisa Darmawahyuni, Muhammad Naufal Rachmatullah, Radiyati Umi Partan, Bambang Tutuko, Ferlita Pratiwi, Andre Herviant Juliano, Rahmi Khoirani, and others. 2020. "Robust Detection of Atrial Fibrillation from Short-Term Electrocardiogram Using Convolutional Neural Networks." Future Generation Computer Systems.

Nurmaini, Siti, Radiyati Umi Partan, Wahyu Caesarendra, Tresna Dewi, Muhammad Naufal Rahmatullah, Annisa Darmawahyuni, Vicko Bhayyu, and Firdaus Firdaus. 2019. "An Automated ECG Beat Classification System Using Deep Neural Networks with an Unsupervised Feature Extraction Technique.” Applied Sciences 9(14):2921.

O’Gara, Patrick T., Frederick G. Kushner, Deborah D. Ascheim, Donald E. Casey, Mina K. Chung, James A. de Lemos, Steven M. Ettinger, James C. Fang, Francis M. Fesmire, Barry A. Franklin, Christopher B. Granger, Harlan M. Krumholz, Jane A. Linderbaum, David A. Morrow, L. Kristin Newby, Joseph P. Ornato, Narith Ou, Martha J. Radford, Jacqueline E. Tamis-Holland, Carl L. Tommaso, Cynthia M. Tracy, Y. Joseph Woo, and David X. Zhao. 2013. "2013 ACCF/AHA Guideline for the Management of ST-Elevation Myocardial Infarction." Journal of the American College of Cardiology 61(4):e78-140.

Oh, Shu Lih, Eddie Y. K. Ng, Ru San Tan, and U. Rajendra Acharya. 2018. "Automated Diagnosis of Arrhythmia Using Combination of CNN and LSTM Techniques with Variable Length Heart Beats." Computers in Biology and Medicine 102:278-87.

Qin, Qin, Jianqing Li, Li Zhang, Yinggao Yue, and Chengyu Liu. 2017. "Combining LowDimensional Wavelet Features and Support Vector Machine for Arrhythmia Beat 
627

628

629

630

631

632

633

634

635

636

637

638

639

640

641

642

643

644

645

646

647

648

649

650

651

652

653

654

655

656

657

658

659

660

661

662

663

664

Classification.” Scientific Reports 7(1):6067.

Rajkumar, A., M. Ganesan, and R. Lavanya. 2019. "Arrhythmia Classification on ECG Using Deep Learning.” Pp. 365-69 in 2019 5th International Conference on Advanced Computing I\& Communication Systems (ICACCS).

Sameni, Reza, Mohammad B. Shamsollahi, Christian Jutten, and Gari D. Clifford. 2007. "A Nonlinear Bayesian Filtering Framework for ECG Denoising.” IEEE Transactions on Biomedical Engineering 54(12):2172-85.

Siontis, Konstantinos C., Peter A. Noseworthy, Zachi I. Attia, and Paul A. Friedman. 2021. "Artificial Intelligence-Enhanced Electrocardiography in Cardiovascular Disease Management." Nature Reviews Cardiology 18(7):465-78.

Tracey, Brian H. and Eric L. Miller. 2012. "Nonlocal Means Denoising of ECG Signals." IEEE Transactions on Biomedical Engineering 59(9):2383-86.

Tutuko, Bambang, Siti Nurmaini, Alexander Edo Tondas, Muhammad Naufal Rachmatullah, Annisa Darmawahyuni, Ria Esafri, Firdaus Firdaus, and Ade Iriani Sapitri. 2021. "AFibNet: An Implementation of Atrial Fibrillation Detection With Convolutional Neural Network."

Walsh, Joseph A., Eric J. Topol, and Steven R. Steinhubl. 2014. "Novel Wireless Devices for Cardiac Monitoring." Circulation 130(7):573-81.

Wang, Jianhong, Yongqiang Ye, Xiang Pan, and Xudong Gao. 2015. "Parallel-Type Fractional Zero-Phase Filtering for ECG Signal Denoising." Biomedical Signal Processing and Control 18:36-41.

Wang, Jibin. 2020. "A Deep Learning Approach for Atrial Fibrillation Signals Classification Based on Convolutional and Modified Elman Neural Network." Future Generation Computer Systems 102(September):670-79.

Wang, Kyuhyun, Richard W. Asinger, and Henry J. L. Marriott. 2003. "ST-Segment Elevation in Conditions Other Than Acute Myocardial Infarction." New England Journal of Medicine 349(22):2128-35.

World Health Organization. 2016. "WHO The Global Burden of Disease."

Yadav, Om Prakash and Shashwati Ray. 2016. "Smoothening and Segmentation of Ecg Signals Using Total Variation Denoising--Minimization-Majorization and Bottom-up Approach." Procedia Computer Science 85:483-89.

Y1ldırım, Özal, Pławiak, Ru-San Tan, U. Rajendra Acharya. 2018. "Arrhythmia Detection Using Deep Convolutional Neural Network With Long Duration ECG Signals." Comput. Biol. Med. 102: 411-420.

Yildirim, Özal. 2018. "A Novel Wavelet Sequence Based on Deep Bidirectional LSTM Network Model for ECG Signal Classification.” Computers in Biology and Medicine 96:189-202.

Yildirim, Ozal, Ulas Baran Baloglu, Ru-San Tan, Edward J. Ciaccio, and U. Rajendra Acharya. 2019. "A New Approach for Arrhythmia Classification Using Deep Coded Features and LSTM Networks." Computer Methods and Programs in Biomedicine 176:121-33.

Peer) Comput. Sci. reviewing PDF | (CS-2021:07:64082:2:0:NEW 22 Nov 2021) 
665 Yildirim, Ozal, Muhammed Talo, Edward J. Ciaccio, Ru San Tan, and U. Rajendra Acharya. 666 2020. "Accurate Deep Neural Network Model to Detect Cardiac Arrhythmia on More than 667 10,000 Individual Subject ECG Records." Computer Methods and Programs in 668 Biomedicine 197:105740.

669 Zubair, Muhammad, Jinsul Kim, and Changwoo Yoon. 2016. "An Automated ECG Beat 670 671 Classification System Using Convolutional Neural Networks.” Pp. 1-5 in 2016 6th international conference on IT convergence and security (ICITCS).

672 
Figure 1

Nine-class of ECG-based rhythm classification
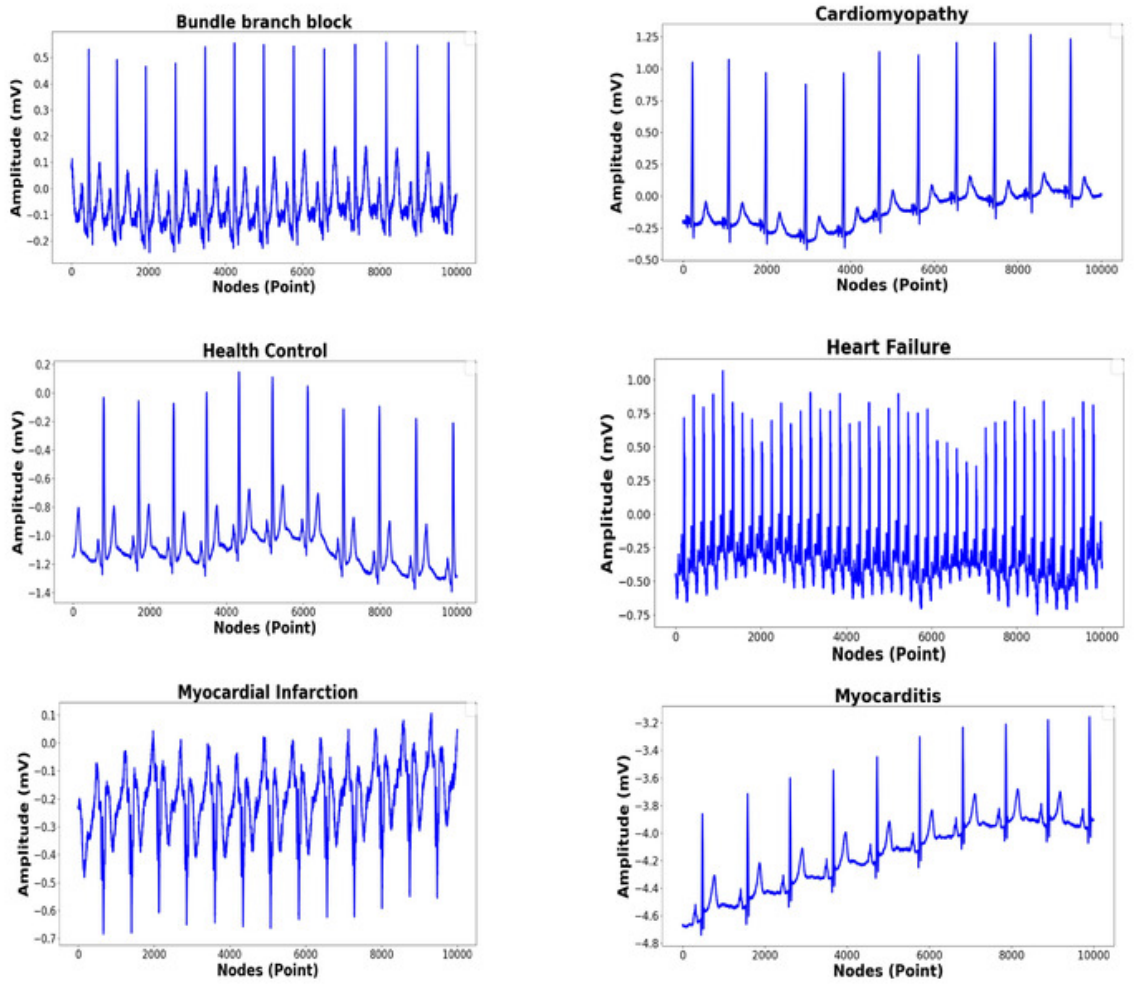
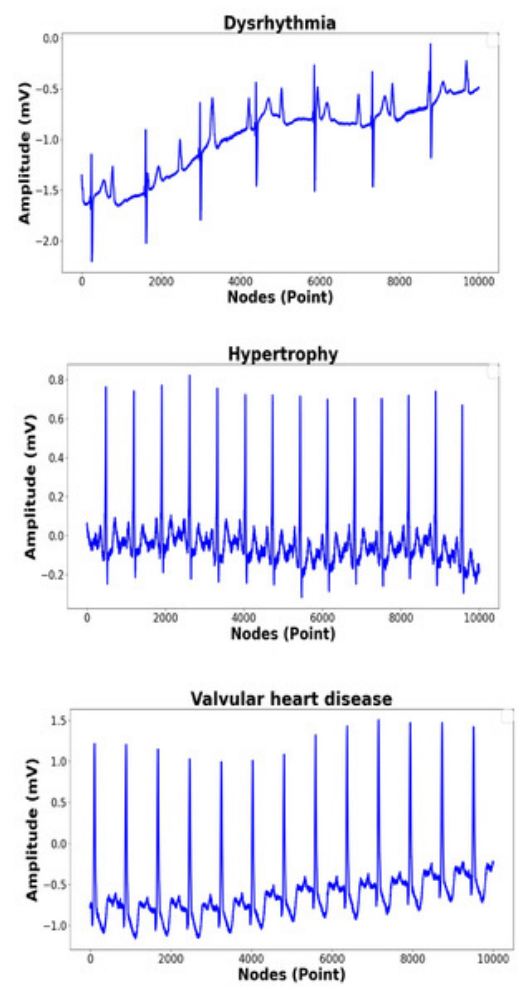
Figure 2

15-class of ECG-based beat classification 


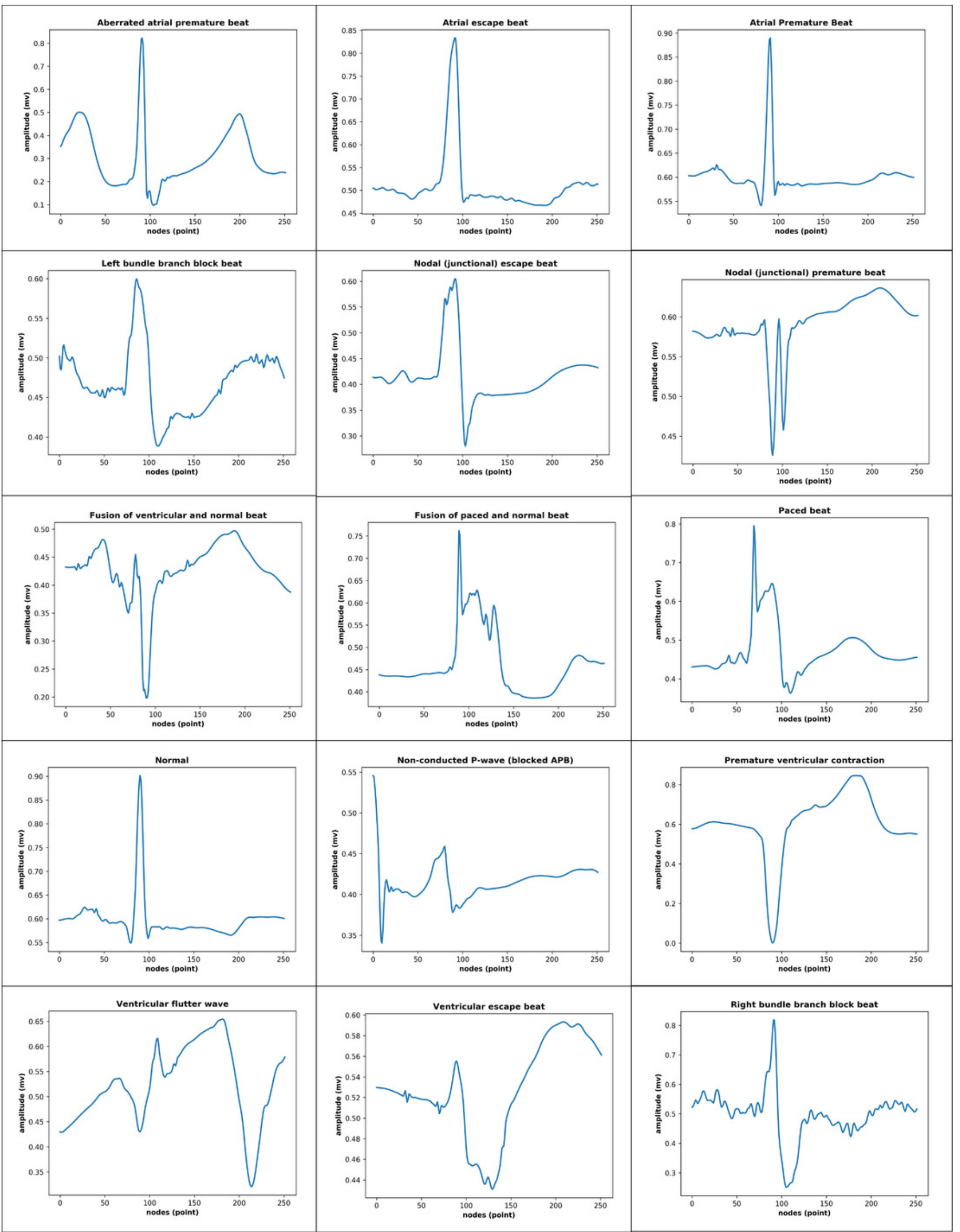


Figure 3

The proposed research methodology of ECG rhythm and beat classification

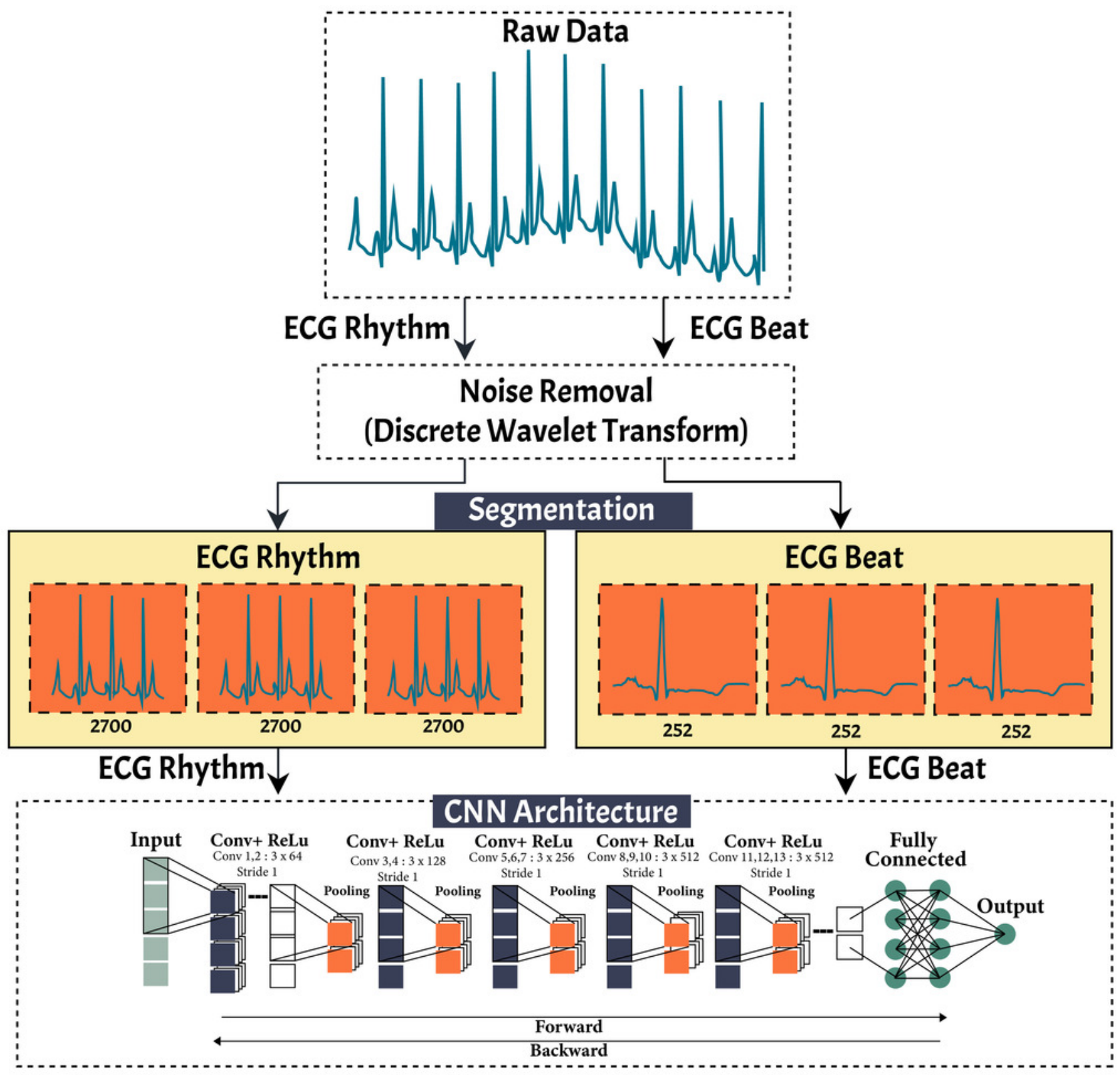


Figure 4

ECG signal segmentation (A) Segmented in 2700 nodes for rhythm feature (B) Segmented in 252 nodes for beat feature 
ECG rhythm segmentation

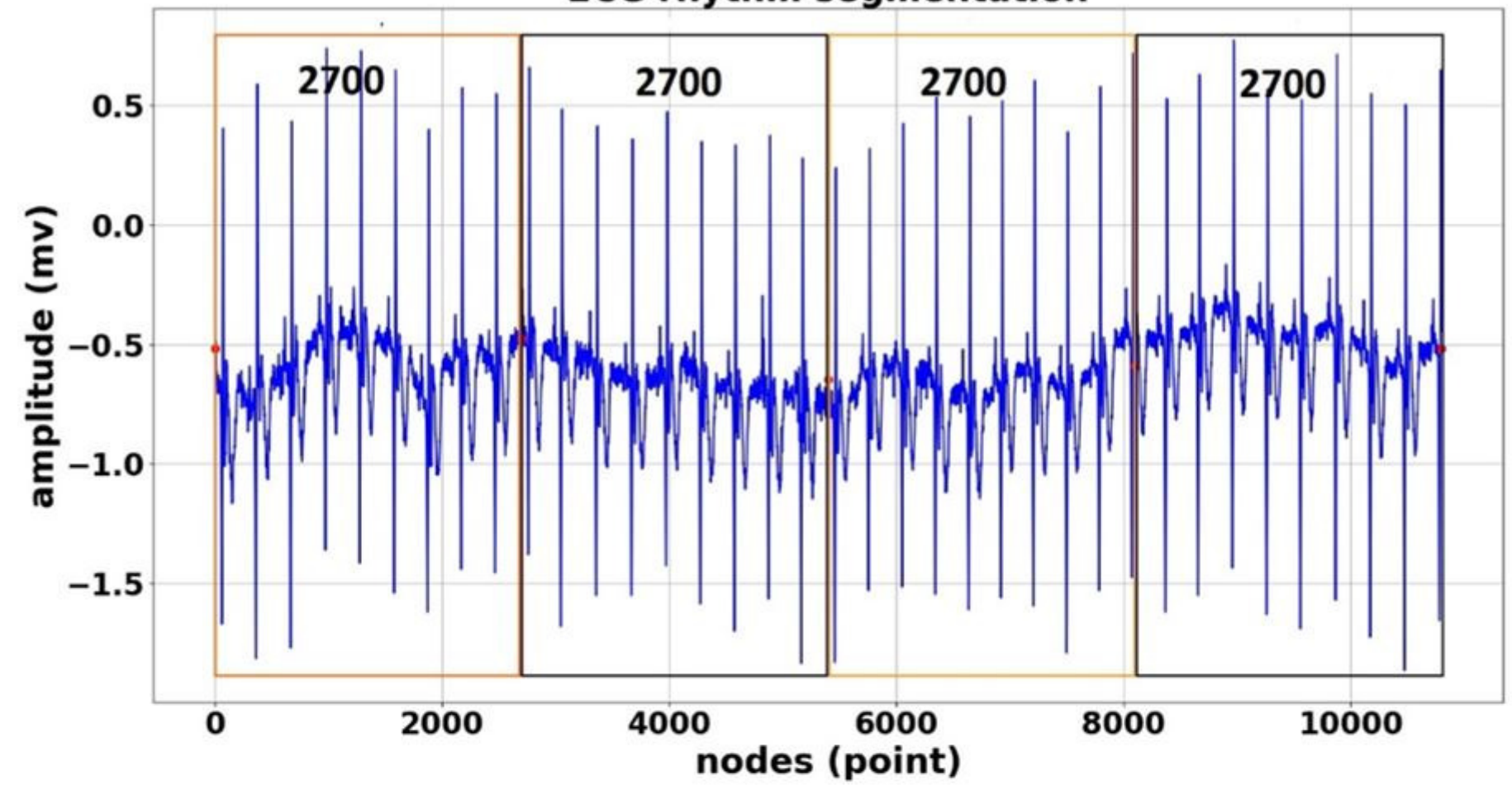

A

ECG beat segmentation

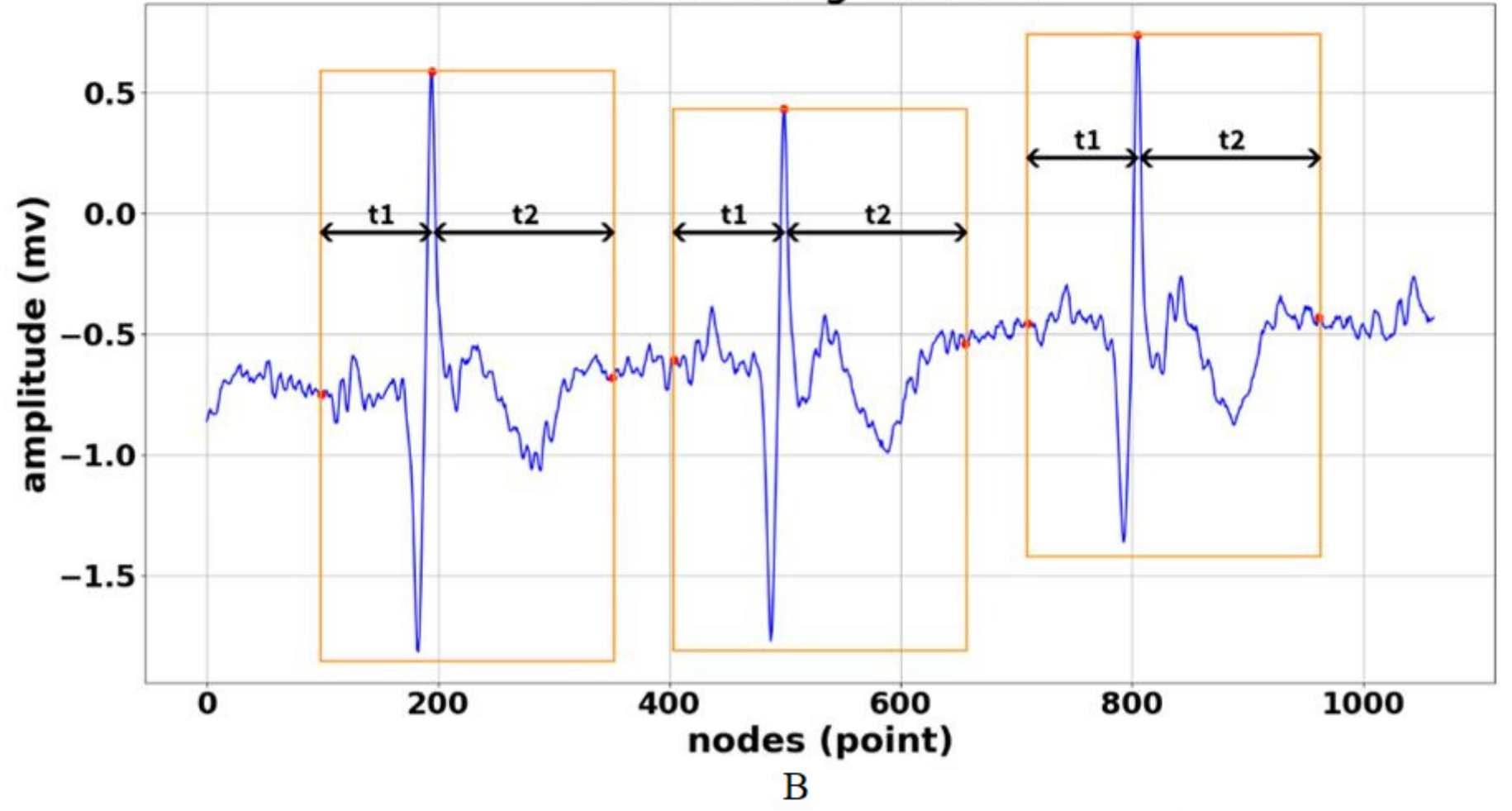


Figure 5

Boxplot of the 10-fold cross-validation results for ECG rhythm classification

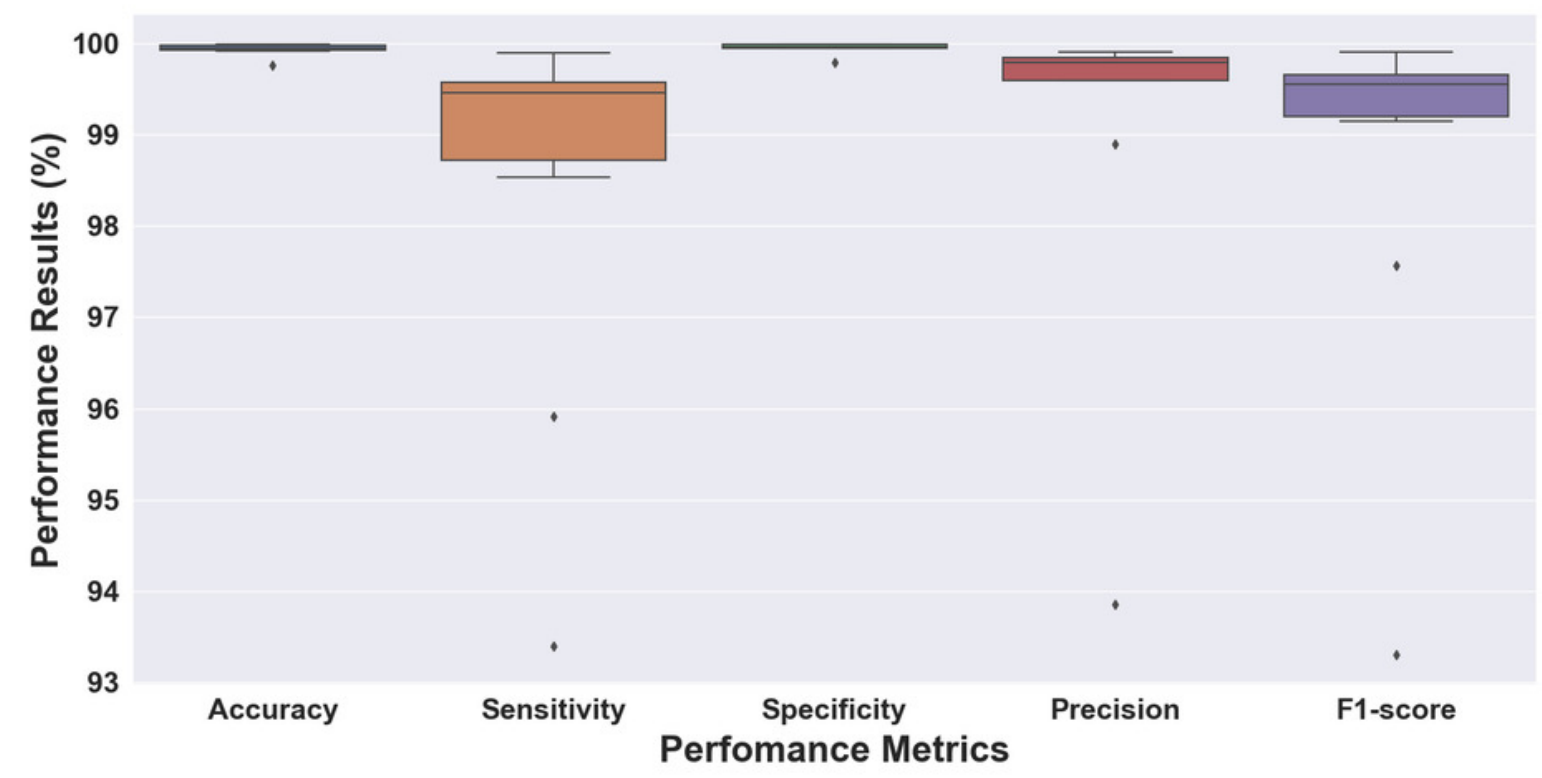




\section{Figure 6}

Confusion matrix evaluation for ECG rhythm classification on validation result

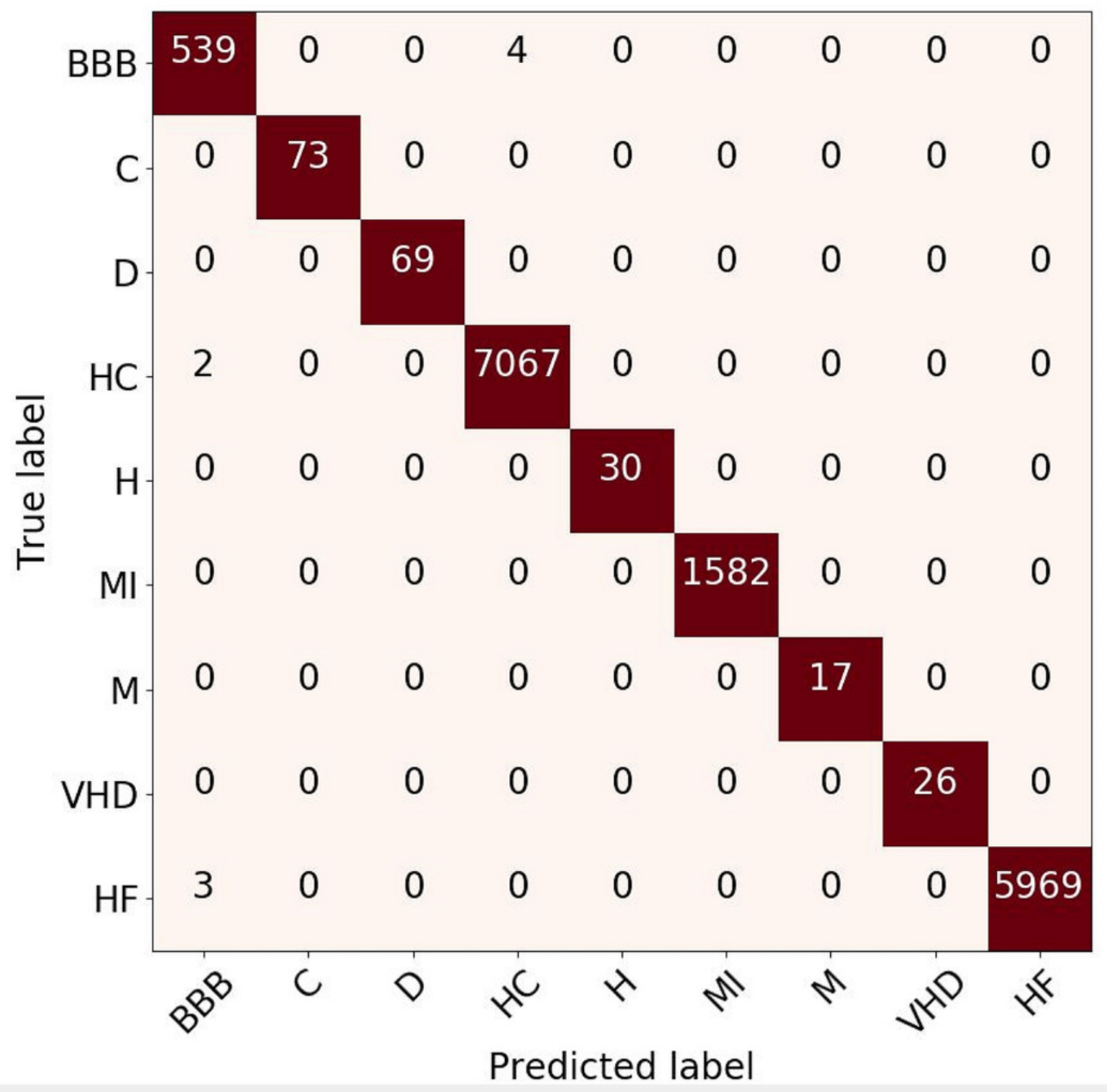


Figure 7

(A) ROC and (B) P-R curves for ECG rhythm classification on the validation result

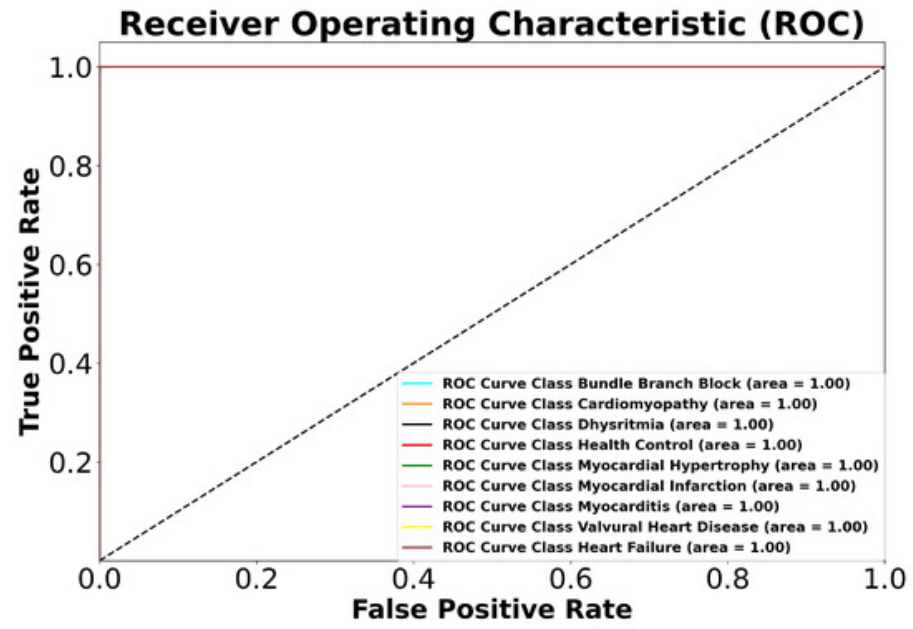

A

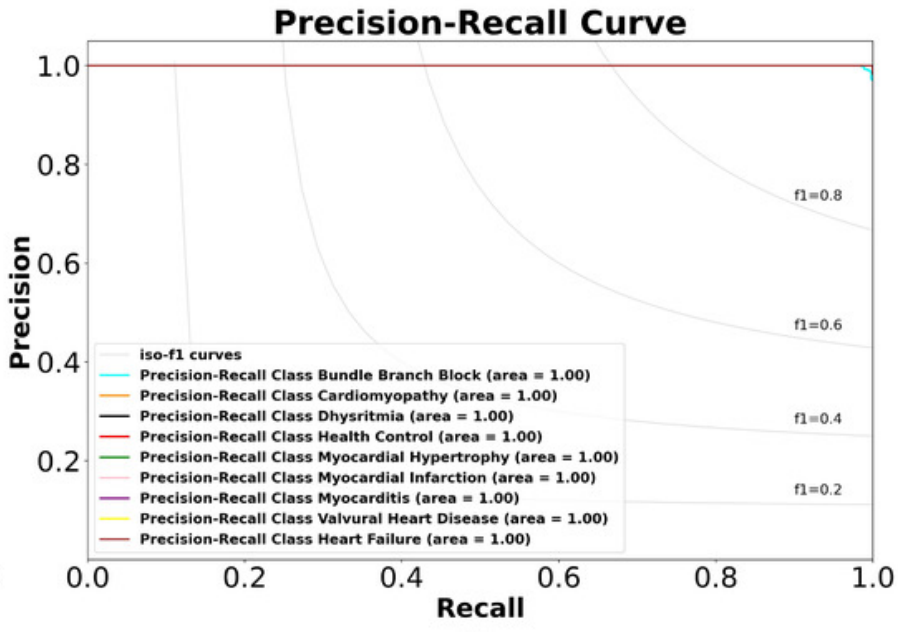

B 


\section{Figure 8}

Boxplot of the 10-fold cross-validation results for the ECG beat classifiation

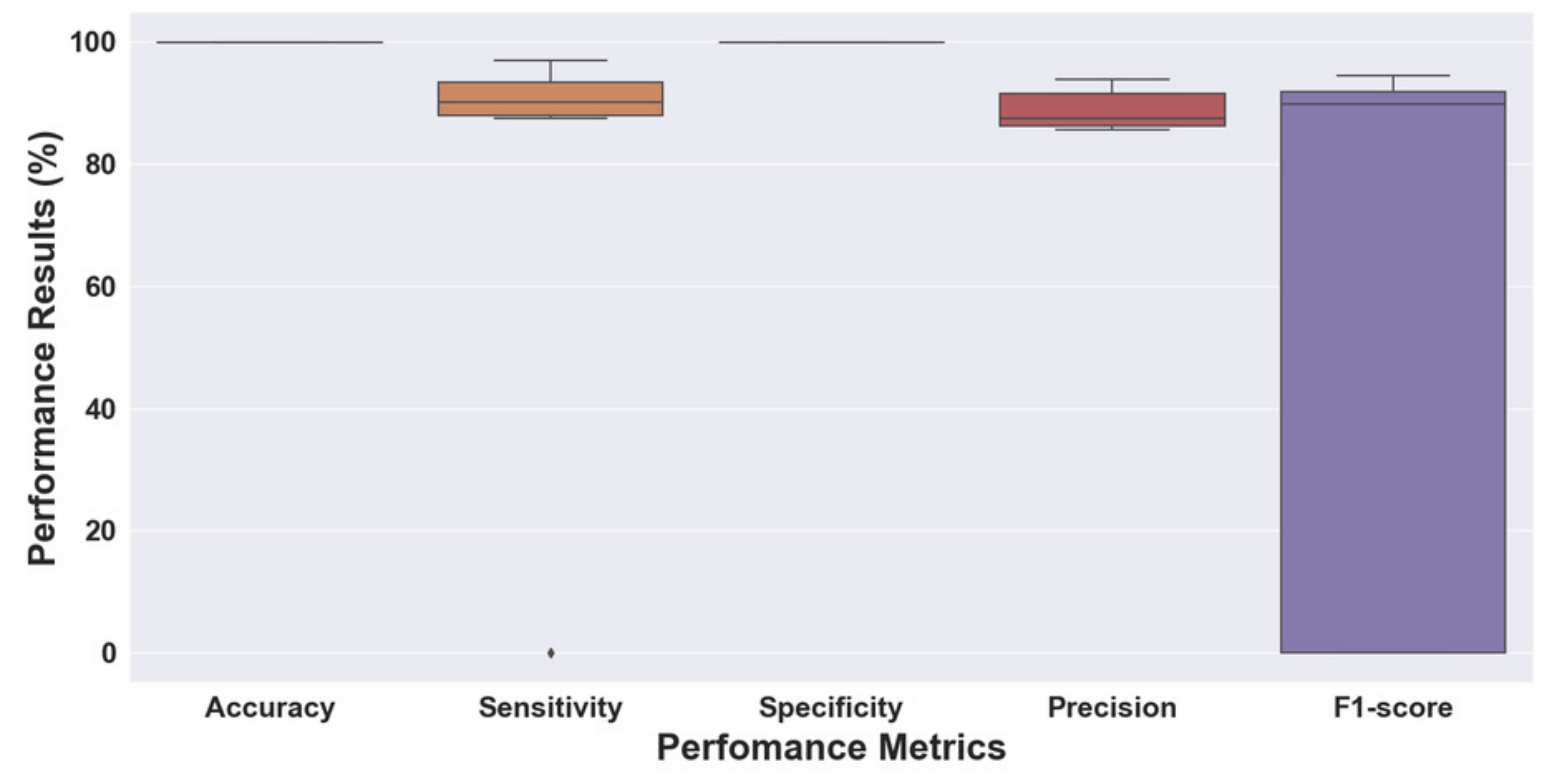




\section{Figure 9}

Confusion matrix evaluation for ECG beat classification on validation result

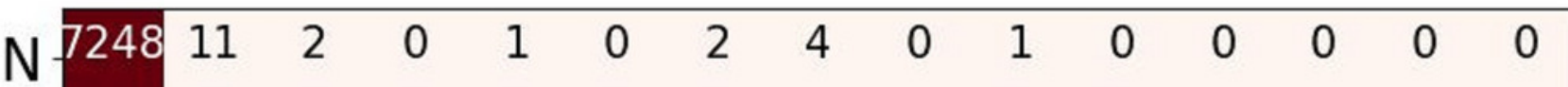

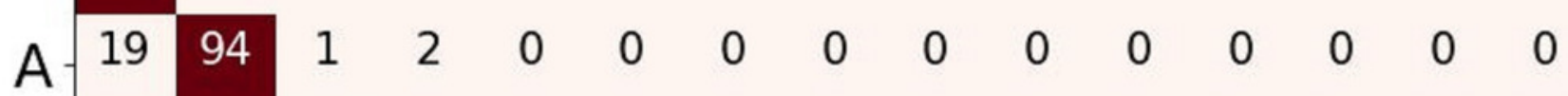

V. $\begin{array}{lllllllllllllll}7 & 0 & 611 & 0 & 0 & 1 & 1 & 1 & 0 & 0 & 0 & 0 & 0 & 0 & 0\end{array}$

R- $\begin{array}{lllllllllllllll}0 & 1 & 0 & 532 & 0 & 0 & 0 & 0 & 0 & 0 & 0 & 0 & 0 & 0 & 0\end{array}$

L- $3 \begin{array}{lllllllllllllll}3 & 0 & 2 & 0 & 553 & 0 & 0 & 0 & 0 & 0 & 0 & 0 & 0 & 0 & 0\end{array}$

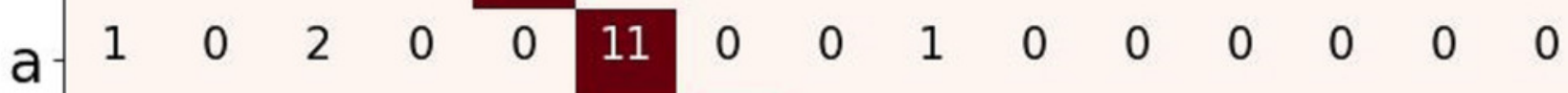

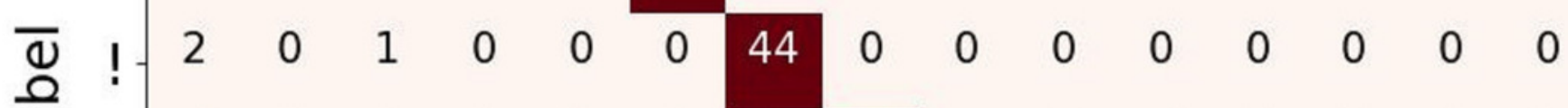

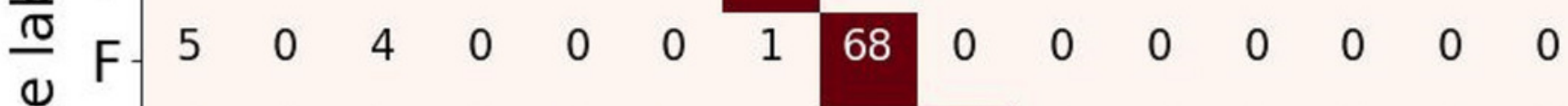

$\stackrel{f}{\perp} \cdot \begin{array}{lllllllllllllll}0 & 0 & 0 & 0 & 0 & 0 & 0 & 0 & 91 & 0 & 0 & 1 & 0 & 0 & 0\end{array}$

j. $6 \begin{array}{lllllllllllllll}6 & 0 & 0 & 0 & 0 & 0 & 0 & 0 & 0 & 16 & 0 & 0 & 0 & 0 & 0\end{array}$

J. $\begin{array}{lllllllllllllll}0 & 0 & 0 & 0 & 0 & 0 & 0 & 0 & 0 & 0 & 6 & 0 & 0 & 0 & 0\end{array}$

P. $\begin{array}{lllllllllllllll}1 & 0 & 0 & 0 & 0 & 0 & 0 & 0 & 0 & 0 & 0 & 499 & 0 & 0 & 0\end{array}$

E- $\begin{array}{lllllllllllllllll}0 & 0 & 1 & 0 & 0 & 0 & 0 & 0 & 0 & 0 & 0 & 0 & 9 & 0 & 0\end{array}$

$\begin{array}{llllllllllllllll}\mathrm{X}-2 & 0 & 0 & 0 & 0 & 0 & 0 & 0 & 0 & 0 & 0 & 0 & 0 & 18 & 0\end{array}$

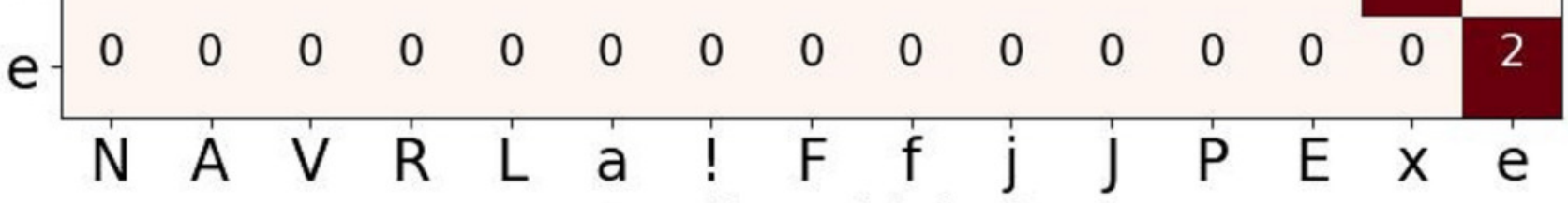

Predicted label 


\section{Figure 10}

\section{(A) ROC and (B) P-R curves for ECG beat classification on the validation result}

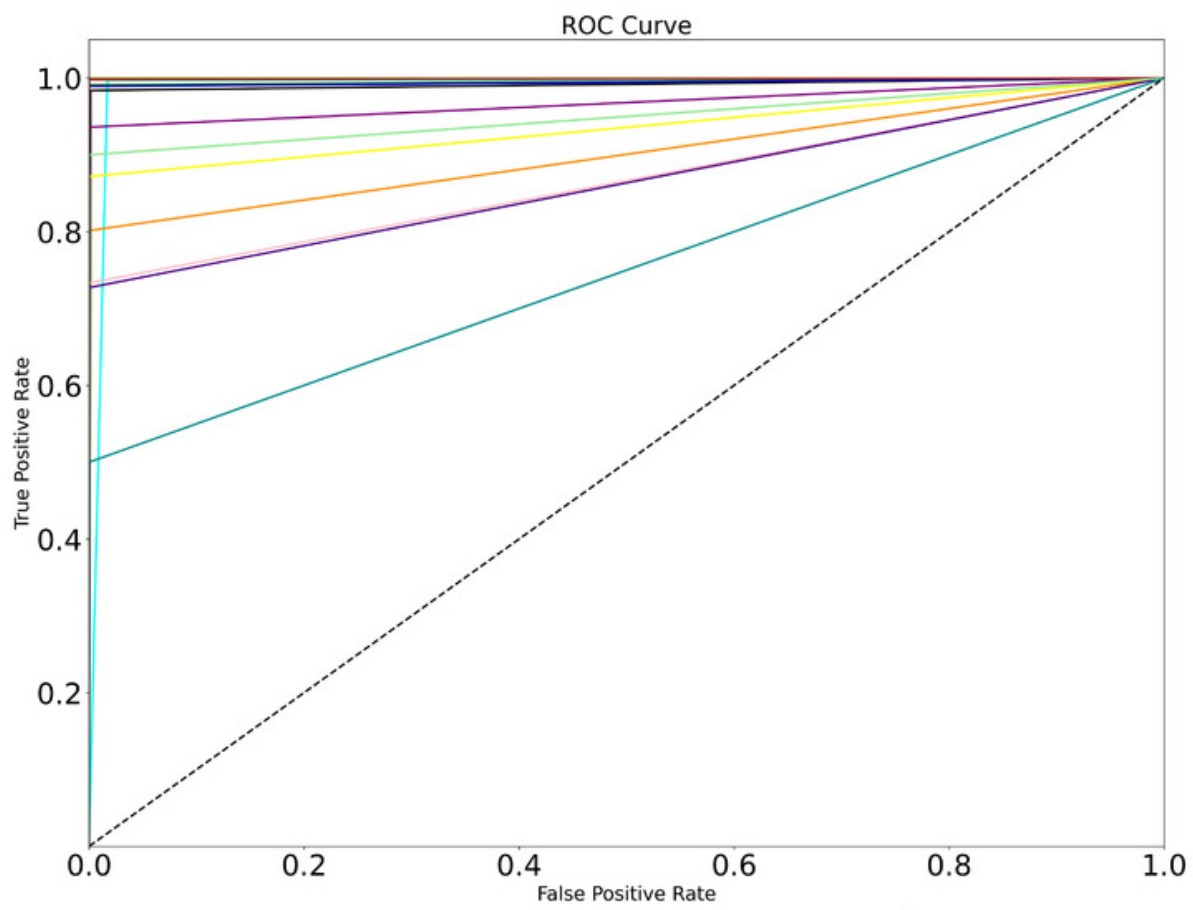

ROC Curve $(\mathrm{N})$ Beat (area $=0.99$ )

ROC Curve (A) Beat (area $=0.90$ )

ROC Curve $(V)$ Beat (area $=0.99$ )

ROC Curve $(R)$ Beat (area $=1.00$ )

ROC Curve $(L)$ Beat (area $=1.00$ )

ROC Curve (a) Beat (area $=0.87$ )

ROC Curve (!) Beat (area $=0.97$ )

ROC Curve $(F)$ Beat (area $=0.94$ )

ROC Curve (f) Beat (area $=0.99$ )

ROC Curve $(\mathrm{j})$ Beat (area $=0.86$ )

ROC Curve (J) Beat (area $=1.00$ )

ROC Curve $(P)$ Beat (area $=1.00)$

ROC Curve (E) Beat (area $=0.95$ )

ROC Curve $(x)$ Beat (area $=0.95$ )

ROC Curve (e) Beat (area $=0.75$ )

\section{$\mathbf{A}$}

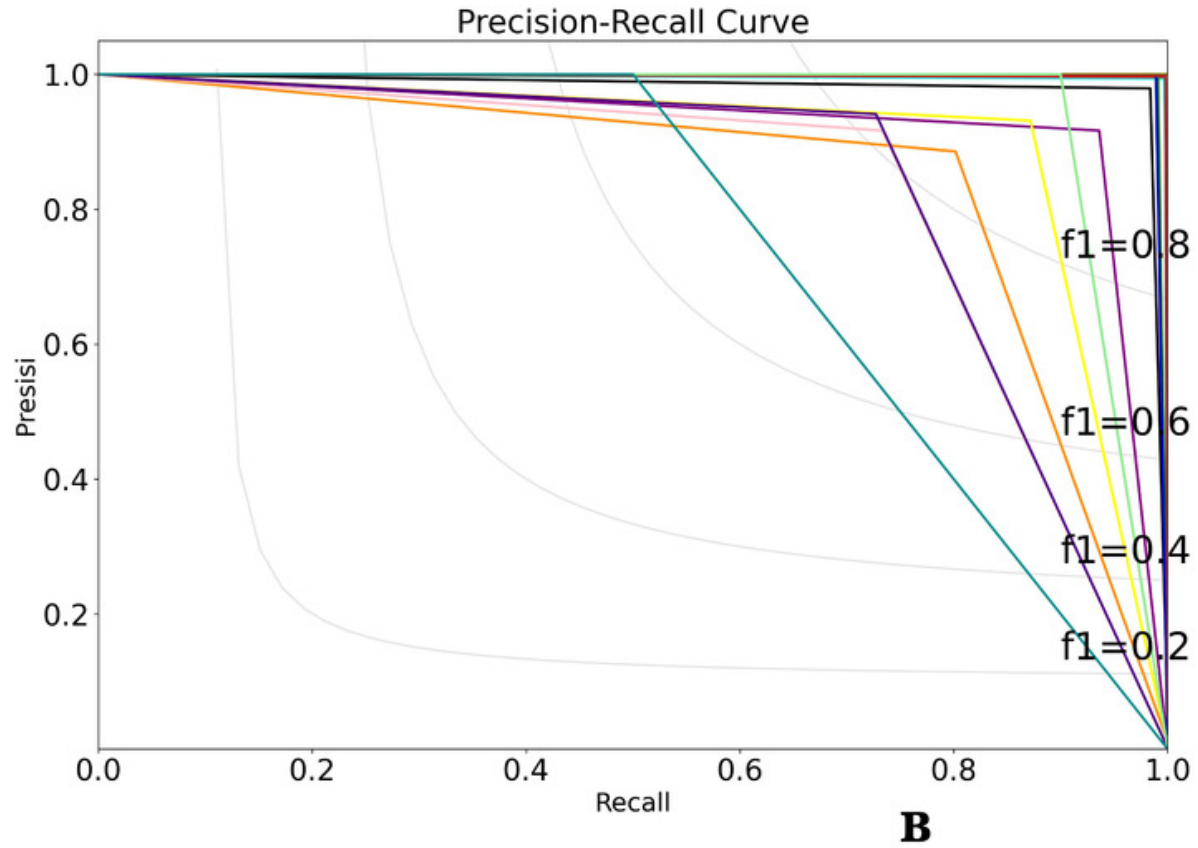

iso-f1 curves

Precision-Recall $(\mathrm{N})$ Beat (area $=0.99)$

Precision-Recall (A) Beat (area $=0.71$ )

Precision-Recall $(\mathrm{V})$ Beat (area $=0.96$ )

Precision-Recall $(R)$ Beat (area $=0.99$ )

Precision-Recall $(\mathrm{L})$ Beat (area $=0.99$ )

Precision-Recall (a) Beat (area $=0.67$ )

Precision-Recall (!) Beat (area $=0.86$ )

Precision-Recall $(F)$ Beat (area $=0.81$ )

Precision-Recall (f) Beat (area $=0.99$ )

Precision-Recall $(\mathrm{j})$ Beat (area $=0.69$ )

Precision-Recall (J) Beat (area $=1.00$ )

Precision-Recall $(\mathrm{P})$ Beat (area $=1.00)$

Precision-Recall $(E)$ Beat (area $=0.90)$

Precision-Recall $(x)$ Beat (area $=0.90$ )

Precision-Recall (e) Beat (area $=0.50$ ) 


\section{Table $\mathbf{1}$ (on next page)}

ECG rhythm data description 
1

2 Table 1. ECG rhythm data description

\begin{tabular}{llll}
\hline Dataset & Class & $\begin{array}{l}\text { Label/ } \\
\text { Abbreviation }\end{array}$ & Records \\
\hline PTB Diagnostic ECG & Bundle Branch Block & BBB & 17 \\
& Cardiomyopathy & C & 17 \\
& Dysrhythmia & D & 16 \\
& Health Control & HC & 80 \\
& Myocardial Hypertrophy & H & 7 \\
& Myocardial Infarction & NU & 368 \\
& Myocarditis & M & 4 \\
BIDMC Congestive Heart & Valvural & VHD & 6 \\
Failure & Congestive Heart Failure & HF & 10 \\
China Physiological Signal & Left bundle branch block & BBB & 207 \\
Challenge 2018 & Right bundle branch & & 1,695 \\
& block & & \\
MIT-BIH Normal Sinus & Normal sinus (healthy & HC & 18 \\
Rhythm & control) & & \\
\hline
\end{tabular}

3 


\section{Table 2 (on next page)}

ECG beat data description 
1

2 Table 2. ECG beat data description

\begin{tabular}{lll}
\hline Dataset & Class & Total Beats \\
\hline MIT-BIH Arrhythmia & Normal Beat (N) & 75,022 \\
& Atrial Premature Beat (A) & 2,546 \\
& Premature Ventricular Contraction (V) & 7,129 \\
& Right Bundle Branch Block Beat (R) & 7,255 \\
& Left bundle branch block beat (L) & 8,072 \\
& Aberrated atrial premature beat (a) & 150 \\
Ventricular flutter wave (!) & 472 \\
& Fusion of ventricular and normal beat & 802 \\
& (F) & \\
& Fusion of paced and normal beat (f) & 982 \\
& Nodal (junctional) escape beat (j) & 229 \\
& Nodal (junctional) premature beat (J) & 83 \\
& Paced beat (/) & 7,025 \\
& Ventricular escape beat (E) & 106 \\
& Non-conducted P-wave (x) & 193 \\
Atrial escape beat (e) & 16 \\
\hline
\end{tabular}

3 


\section{Table 3(on next page)}

The total episodes after segmentation of 2700 nodes 
1

2 Table 3. The total episodes after segmentation of 2700 nodes

\begin{tabular}{|c|c|c|c|c|}
\hline \multirow[t]{2}{*}{ Dataset } & \multirow[t]{2}{*}{ Class } & \multicolumn{2}{|c|}{$\begin{array}{l}\text { Total rhythm after segmentation } \\
\text { of } 2700 \text { nodes (episode) }\end{array}$} & \multirow[t]{2}{*}{$\begin{array}{l}\text { Unseen } \\
\text { Set }\end{array}$} \\
\hline & & Training Set & Validation Set & \\
\hline \multirow[t]{8}{*}{ PTB Diagnostics ECG } & $\mathrm{BBB}$ & 230 & 26 & \multirow{8}{*}{ - } \\
\hline & $\mathrm{C}$ & 658 & 73 & \\
\hline & $\mathrm{D}$ & 619 & 69 & \\
\hline & $\mathrm{HC}$ & 3,096 & 344 & \\
\hline & $\mathrm{H}$ & 271 & 30 & \\
\hline & MI & 14,242 & 1,582 & \\
\hline & $\mathrm{M}$ & 155 & 17 & \\
\hline & VHD & 232 & 26 & \\
\hline BIDMC Congestive Heart Failures & $\mathrm{HF}$ & 53,738 & 5,969 & 6,647 \\
\hline China Physiological Signal & BBB & & & \\
\hline Challenge 2018 & $\mathrm{BBB}$ & 4,651 & 514 & 614 \\
\hline MIT-BIH Normal Sinus Rhythm & $\mathrm{HC}$ & 60,523 & 6,723 & 7,423 \\
\hline Total & & 138,415 & 15,373 & 14,684 \\
\hline
\end{tabular}




\section{Table 4 (on next page)}

Performance results of the nine class with ECG rhythm in the intra-patient scheme 
1

2 Table 4. Performance results of the nine class with ECG rhythm in the intra-patient scheme

\begin{tabular}{|c|c|c|c|c|c|c|c|c|c|c|}
\hline \multirow{2}{*}{$\begin{array}{l}\text { Performance } \\
\text { Metrics (\%) }\end{array}$} & \multicolumn{9}{|l|}{ Class } & \multirow[t]{2}{*}{ Average } \\
\hline & $\mathrm{BBB}$ & $\mathrm{C}$ & $\mathrm{D}$ & $\mathrm{HC}$ & $\mathrm{H}$ & MI & M & VHD & $\mathrm{HF}$ & \\
\hline Accuracy & 99.94 & 100 & 100 & 99.96 & 100 & 100 & 100 & 100 & 99.98 & 99.98 \\
\hline Sensitivity & 99.26 & 100 & 100 & 99.97 & 100 & 100 & 100 & 100 & 99.95 & 99.90 \\
\hline Specificity & 99.08 & 100 & 100 & 99.94 & 100 & 100 & 100 & 100 & 100.00 & 99.89 \\
\hline Precision & 99.17 & 100 & 100 & 99.96 & 100 & 100 & 100 & 100 & 99.97 & 99.90 \\
\hline F1-Score & 99.97 & 100 & 100 & 99.98 & 100 & 100 & 100 & 100 & 99.97 & 99.99 \\
\hline
\end{tabular}

3

4

5

6

7

8 


\section{Table 5 (on next page)}

The performance results of the 15-class with ECG beats in the intra-patient scheme 
1

2 Table 5. The performance results of the 15-class with ECG beats in the intra-patient scheme Beats Class Performance Results (\%)

\begin{tabular}{llllll} 
& Accuracy & Senisitivity & Specificity & Precision & F1-Score \\
\hline $\mathrm{N}$ & 99.32 & 99.36 & 99.19 & 99.71 & 99.53 \\
$\mathrm{~A}$ & 99.65 & 88.67 & 99.77 & 81.03 & 84.68 \\
$\mathrm{~V}$ & 99.76 & 97.91 & 99.89 & 98.38 & 98.15 \\
$\mathrm{R}$ & 99.96 & 99.62 & 99.98 & 99.81 & 99.71 \\
$\mathrm{~L}$ & 99.93 & 99.81 & 99.94 & 99.10 & 99.46 \\
$\mathrm{a}$ & 99.94 & 91.66 & 99.95 & 73.33 & 81.48 \\
! & 99.92 & 91.66 & 99.96 & 93.61 & 92.63 \\
$\mathrm{~F}$ & 99.84 & 93.15 & 99.89 & 87.17 & 90.06 \\
$\mathrm{f}$ & 99.97 & 98.91 & 99.98 & 98.91 & 98.91 \\
$\mathrm{j}$ & 99.92 & 94.11 & 99.93 & 72.72 & 82.05 \\
$\mathrm{~J}$ & 100 & 100 & 100 & 100 & 100 \\
$\mathrm{P}$ & 99.97 & 99.8 & 99.98 & 99.8 & 99.8 \\
$\mathrm{E}$ & 99.98 & 100 & 99.98 & 90 & 94.73 \\
$\mathrm{X}$ & 99.97 & 100 & 99.97 & 90 & 94.73 \\
$\mathrm{e}$ & 100 & 100 & 100 & 100 & 100 \\
Average & 99.87 & 96.97 & 99.89 & 92.23 & 94.39 \\
\hline
\end{tabular}

3 


\section{Table 6(on next page)}

Performance results of the inter-patient scheme 
1

2 Table 6. Performance results of the inter-patient scheme

\begin{tabular}{llllll}
\hline \multirow{2}{*}{ Performance Metrics (\%) } & \multicolumn{5}{l}{ Class } \\
\cline { 2 - 6 } & BBB & HC & HF & V & L \\
\hline Accuracy & 99.97 & 99.99 & 99.98 & 97.05 & 99.53 \\
Sensitivity & 98.91 & 100.0 & 100.0 & 96.19 & 99.00 \\
Specificity & 100.0 & 99.98 & 99.97 & 97.13 & 99.68 \\
Precision & 99.45 & 99.99 & 99.98 & 84.98 & 98.88 \\
F1-Score & 99.97 & 100.0 & 100.0 & 84.27 & 98.94 \\
\hline
\end{tabular}

3 


\section{Table 7 (on next page)}

Comparison results and DL algorithms between the related work and our proposed method in this work 
1 Table 7. Comparison results with the state of the art

\begin{tabular}{|c|c|c|c|c|c|c|c|}
\hline \multirow[t]{2}{*}{ Authors } & \multirow[t]{2}{*}{ Class } & \multirow[t]{2}{*}{ Feature } & \multirow[t]{2}{*}{ Method } & \multicolumn{4}{|c|}{ Performance Results (\%) } \\
\hline & & & & Acc. & Sens. & Spec. & Pre. \\
\hline $\begin{array}{l}\text { Rajkumar et al. } \\
2019\end{array}$ & 8 & rhythm & 1D-CNN & 93.60 & - & - & - \\
\hline $\begin{array}{l}\text { Yildirim et al. } \\
2018\end{array}$ & 17 & rhythm & $1 \mathrm{D}-\mathrm{CNN}$ & 91.30 & 83.90 & - & 85.4 \\
\hline $\begin{array}{l}\text { Nannavecchia et } \\
\text { al. } 2021\end{array}$ & 21 & beat & 1D-CNN & 89.51 & 87.79 & - & 86.78 \\
\hline $\begin{array}{l}\text { Yildirim et al. } \\
2019\end{array}$ & 5 & rhythm & LSTM & 99.23 & - & - & 99.00 \\
\hline Gao et al. 2019 & 8 & rhythm & LSTM & 99.26 & - & 99.26 & 99.14 \\
\hline $\begin{array}{l}\text { Lui and Chow } \\
2018\end{array}$ & 4 & beat & 1D-CNN-LSTM & - & 92.40 & 97.70 & - \\
\hline $\begin{array}{l}\text { Yildirim et al. } \\
2020\end{array}$ & 7 & beat & 1D-CNN-LSTM & 92.24 & 80.15 & 98.72 & 80.31 \\
\hline Oh et al. 2018 & 5 & rhythm & 1D-CNN-LSTM & 98.10 & - & - & 97.50 \\
\hline Chen et al. 2020 & 6 & rhythm & 1D-CNN-LSTM & 99.32 & 97.75 & - & - \\
\hline Luo et al. 2021 & 9 & rhythm & $\begin{array}{l}\text { 1D-CNN- } \\
\text { LSTM-GRU }\end{array}$ & 99.01 & 99.58 & - & 99.44 \\
\hline Our work & $\begin{array}{l}9 \\
15\end{array}$ & $\begin{array}{l}\text { beat } \\
\text { rhythm }\end{array}$ & $\begin{array}{l}\text { 1D-CNN } \\
1 \mathrm{D}-\mathrm{CNN}\end{array}$ & $\begin{array}{l}99.98 \\
99.87\end{array}$ & $\begin{array}{l}99.90 \\
96.97\end{array}$ & $\begin{array}{l}99.89 \\
99.89\end{array}$ & $\begin{array}{l}99.90 \\
92.23 \\
\end{array}$ \\
\hline
\end{tabular}

2 *Acc. (accuracy); Sen. (sensitivity); Spec. (specificity); Pre, precision 